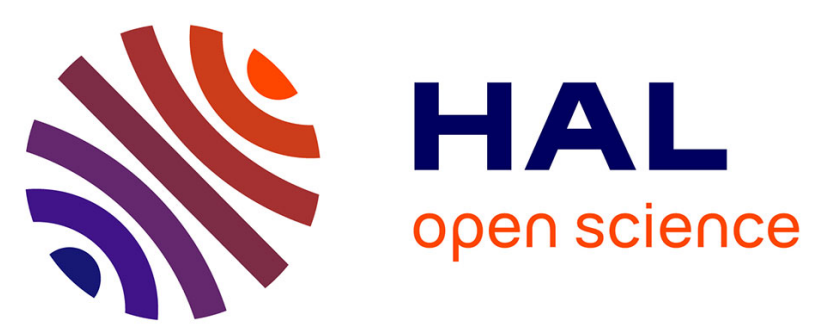

\title{
Bosonization approach to charge and spin dynamics of one-dimensional spin-1/2 fermions with band curvature in a clean quantum wire
}

Sofian Teber

\section{- To cite this version:}

Sofian Teber. Bosonization approach to charge and spin dynamics of one-dimensional spin- $1 / 2$ fermions with band curvature in a clean quantum wire. Physical Review B: Condensed Matter and Materials Physics (1998-2015), 2007, 76 (4), pp.045309. 10.1103/PhysRevB.76.045309 . hal-01697268

\section{HAL Id: hal-01697268 \\ https://hal.science/hal-01697268}

Submitted on 2 Mar 2022

HAL is a multi-disciplinary open access archive for the deposit and dissemination of scientific research documents, whether they are published or not. The documents may come from teaching and research institutions in France or abroad, or from public or private research centers.
L'archive ouverte pluridisciplinaire HAL, est destinée au dépôt et à la diffusion de documents scientifiques de niveau recherche, publiés ou non, émanant des établissements d'enseignement et de recherche français ou étrangers, des laboratoires publics ou privés. 


\title{
Bosonization approach to charge and spin dynamics of one-dimensional spin- $\frac{1}{2}$ fermions with band curvature in a clean quantum wire
}

\author{
Sofian Teber* \\ The Abdus Salam ICTP, Strada Costiera 11, 34014, Trieste, Italy \\ and Institut NEEL, CNRS and Université Joseph Fourier, Boîte Postale 166, 38042, Grenoble, France
}

(Received 5 October 2006; revised manuscript received 16 April 2007; published 9 July 2007)

\begin{abstract}
We consider one-dimensional spin- $\frac{1}{2}$ fermions in a clean quantum wire, with forward scattering interactions and a nonlinear single-particle spectrum $\xi_{k}=v|k|+k^{2} / 2 m$, where $v$ is the Fermi velocity and $1 / m$ is the band curvature. We calculate the dynamical structure factor (DSF) of the model at small wave vector $q$ with the help of the bosonization technique. For spinless fermions, we show that, starting from the single-parametric spectrum $\omega=u|q|$, bosonization emulates the two-parametric excitation spectrum $\omega=u|q| \pm q^{2} / 2 m^{*}$, where $m^{*}$ decreases with increasing repulsive interactions. Moreover, away from the excitation cone, i.e., $\omega \gg u|q|$, bosonization yields the two-pair excitation continuum of the DSF. For spinful fermions, we show that the spin-charge coupling (SCC) due to band curvature affects the charge and spin DSFs in an asymmetric way. For the charge DSF, SCC manifests as a two-peak structure: a charge peak at $\omega=u_{\rho}|q|$ but also a spin peak at $\omega=u_{\sigma}|q|$, as charge fluctuations may decay via chargeless spin-singlet excitations. For the magnetic DSF, SCC manifests as a continuous transfer of magnetic spectral weight to frequencies $\omega>u_{\sigma}|q|$, as spin fluctuations decay via pairs of chargeless spin and spinless charge-neutral excitations.
\end{abstract}

DOI: 10.1103/PhysRevB.76.045309

PACS number(s): 73.21.Hb, 71.10.Pm

\section{INTRODUCTION}

Correlation functions of one-dimensional (1D) fermions in quantum wires are conveniently calculated within the Tomonaga-Luttinger (TL) model and with the help of the bosonization technique. The TL model assumes the linearity of the single-particle spectrum with respect to momentum:

$$
\xi_{k}=v\left(|k|-k_{F}\right) \quad \text { (Tomonaga-Luttinger), }
$$

where $v$ is the Fermi velocity and $k_{F}$ the Fermi momentum, and interactions of the forward scattering type, so-called $g_{2}$ and $g_{4}$ processes in $g$-ology. The forward scattering nature of the interactions implies that the system has no spectral gap whereas the linearity of the spectrum yields Lorentz invariance. These assumptions make the model exactly soluble by mapping the interacting fermions onto free bosonic excitations or plasmons. ${ }^{1-5}$ As a consequence, e.g., transport properties of TL liquids are easily accessed with the help of bosonization (see the recent monographs), ${ }^{6,7}$

Even though the TL model allows a standard description of fermions in quantum wires, as the Landau theory of Fermi liquids allows a standard description of 3D systems, (see Ref. 8 for a review), both assumptions of a relativistic singleparticle spectrum and forward scattering interactions often constitute an oversimplified description of 1D fermionic systems. As a matter of fact, fermions hopping with an amplitude $t$ on a 1D lattice of parameter $a$ have a single-particle spectrum given by

$$
\xi_{k}=-2 t \cos (k a) \quad \text { (Hubbard). }
$$

Adding interactions between such fermions leads to the $1 D$ Hubbard model (see Ref. 9 for a review), one of the most fundamental models of solid-state physics. In the absence of disorder and away from half filling we may assume that the system has no spectral gap, e.g., it is not in a Mott-insulating phase. One may then focus on the low-energy properties of this model by expanding Eq. (2) around the Fermi points $\pm k_{F}$, where the + sign refers to right movers and the - sign to left movers. In the lowest order, this yields the TL model Eq. (1) with $v=2 t a \sin \left(k_{F} a\right)$. Because of the exact solubility of the TL model, the low-energy properties of the Hubbard model are thus known exactly. On the other hand, except for the case of free fermions, which is again exactly soluble, less is known about the high-energy properties of the Hubbard model. Considerable work has been devoted to exact studies related to the nature of its ground state and its low-energy excitations. This has been done with the help of the Bethe ansatz (see Refs. 9 and 10 for reviews), which allows a fully nonperturbative handling of the nonlinear spectrum Eq. (2), and interaction effects. This technique is, however, extremely difficult to generalize to the calculation of correlation functions. Formidable efforts are made to achieve this task but they are limited at present to the half-filled case ${ }^{11}$ and to the $X X Z$ Heisenberg spin chain (see Ref. 12 for a review and Ref. 13 for an application to the present problem). The $X X Z$ spin chain is equivalent to spinless fermions (see Ref. 14 for a review on spin chains and the Hubbard model).

In this contribution we focus on a different approach to the high-energy properties of strongly correlated 1D fermions. This amounts to starting from the TL model, assuming forward scattering interactions, and introducing nonlinear corrections to the linear single-particle spectrum. Restricting ourselves to the lowest-order correction yields

$$
\xi_{k}=v\left(|k|-k_{F}\right)+\left(|k|-k_{F}\right)^{2} / 2 m+O\left(\left(|k|-k_{F}\right)^{3}\right),
$$

where $1 / m=2 t a^{2} \cos \left(k_{F} a\right)$ is the band curvature or, equivalently, $m$ is the band mass. For Galilean-invariant systems, there are no higher-order corrections than the band curvature. On the other hand, for lattice fermions, Eq. (3) neglects an infinite number of irrelevant (in the renormalization group sense) terms. This means that, with respect to the Hubbard model Eq. (2), the model defined by Eq. (3) takes into ac- 
count only the most relevant of these terms which, below half filling, corresponds to the curvature of the band spectrum. Such a simplified model then allows a nonperturbative treatment of band curvature and the extraction of information about dynamical correlation functions of interacting 1D fermions beyond the TL model and toward the Hubbard model.

Historically, such a generalization of the Tomonaga model was first considered by Schick $;^{3}$ see also Refs. 5 and 15. More recently, this model has been considered in relation to the damping of 1D plasmons, ${ }^{13,16-18}$ and the drag resistivity due to forward scattering between quantum wires. ${ }^{19}$ Most of these studies focus on the density-density correlation function in the case of spinless fermions (see Refs. 13 and 17-21) or the equivalent $X X Z$ Heisenberg spin chain (see Refs. 13 and 18). Various techniques have been considered to deal with this problem; mainly, fermionic, ${ }^{17-21}$ field theory, ${ }^{13}$ Bethe ansatz, ${ }^{13}$ and numerical (Density Matrix Renormalization Group). ${ }^{13}$ Nonperturbative approaches to band curvature may be found in Refs. 13, 18, and 19. In this paper we will focus on a field theory (bosonization) approach to the computation of density-density correlation functions and consider the case of fermions with spin.

The paper is structured as follows. In Sec. II we will review some known results (for free as well as interacting) ${ }^{13,18}$ fermions and motivate the need for a bosonization approach. In Sec. III the case of spinless fermions will be considered, and the results compared with the ones found in the literature. ${ }^{13,18}$ In Sec. IV the case of spin$1 / 2$ fermions will be considered following Ref. 15. In Sec. V we will summarize our results and conclude.

\section{MOTIVATIONS FOR A BOSONIZATION APPROACH}

The main object we will consider in the following is the density-density correlation function or polarization operator:

$$
\Pi(x, \tau)=\langle\rho(x, \tau) \rho(0,0)\rangle,
$$

where $\rho$ is the fermion density measured relative to a neutralizing jellium.

For free fermions this correlation function is known exactly with band curvature. It is simply the polarization bubble, i.e., the convolution of two free fermionic Green's functions. In Fourier space it reads

$$
\Pi^{0}(i \omega, q)=\frac{m}{\pi q} \ln \left(\frac{(i \omega)^{2}-\left(\omega_{-}^{0}\right)^{2}}{(i \omega)^{2}-\left(\omega_{+}^{0}\right)^{2}}\right),
$$

where the superscript refers to free fermions and we have taken into account the spin degeneracy. In Eq. (4), the twoparametric family appears:

$$
\omega_{ \pm}^{0}(q)=v q \pm \frac{q^{2}}{2 m} .
$$

In the (particle-hole) excitation spectrum of the system, this family bounds the region of decay of the coherent bosonic excitation, the plasmon which corresponds to a coherent particle-hole pair, into the incoherent continuum of single particle-hole pairs (see Fig. 1). This is best revealed by looking at the dynamic structure factor (DSF) of the fermions,

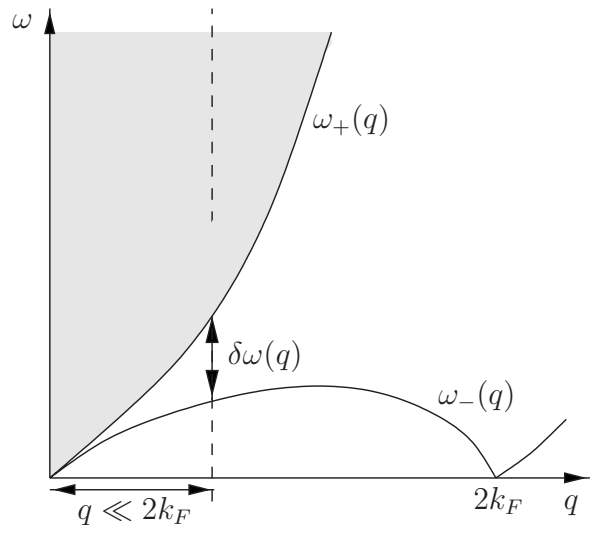

FIG. 1. Schematic view on the spectrum of excitations of 1D spinless fermions. In the case of free fermions, a single-pair excitation continuum $S^{0}$ lies between the $\omega_{-}$and $\omega_{+}$branches. In the presence of forward scattering interactions a multipair excitation continuum $\left(S^{(2)}\right.$ at the two-pair level) spreads above the $\omega_{+}$branch and a divergent spectral weight appears along the $\omega_{-}$branch (cf. Fig. 2).

which corresponds to the dissipative part of the retarded polarization operator:

$$
S(\omega, q)=-\operatorname{Im} \Pi^{R}(\omega, q) .
$$

For free fermions, Eqs. (4) and (6) yield (at $T=0$ )

$$
\begin{gathered}
S^{0}(\omega, q)=\frac{m}{|q|}\left[\theta\left(\frac{m v}{q}\left(\omega-\omega_{-}^{0}\right)\right)-\theta\left(\frac{m v}{q}\left(\omega-\omega_{+}^{0}\right)\right)\right], \\
-\{\omega \rightarrow-\omega\},
\end{gathered}
$$

which, for a fixed momentum $q$, is nonzero and independent of frequency in the range: $\omega_{-}^{0}<\omega<\omega_{+}^{0}$. In particular, at the plasmon frequency, Eq. (7) yields a finite result:

$$
S^{0}(\omega=v q, q)=\frac{m}{|q|} .
$$

Moreover, the spectral width of the DSF yields the inverse lifetime of the bosonic excitations:

$$
\delta \omega^{0}=\left|\omega_{+}^{0}-\omega_{-}^{0}\right|=\frac{q^{2}}{m} .
$$

On the other hand, expanding Eq. (4) in $1 / m$ yields

$$
\Pi^{0}(i \omega, q)=\frac{2}{\pi v} \sum_{n=0}^{\infty} \frac{1}{2 n+1}\left(\frac{q^{2}}{m}\right)^{2 n} \frac{(v q)^{2 n+2}}{\left[(i \omega)^{2}-(v q)^{2}\right]^{2 n+1}},
$$

which reveals a basic difference with respect to the exact result of Eq. (4): order by order the dissipative part is singular at the plasmon frequency $\omega=v|q|$, with $\delta$-function singularities. In particular, within the TL approximation $(m \rightarrow \infty)$,

$$
S_{T L}^{0}(\omega, q)=|q| \delta[\omega-v|q|],
$$

which implies that plasmons have an infinite lifetime, i.e., are free. The divergency of the DSF, in the limit $m \rightarrow \infty$, 


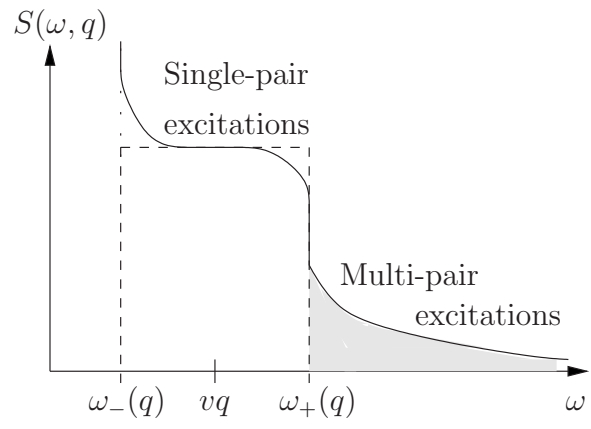

FIG. 2. Schematic view of the dynamical structure factor of interacting spinless fermions as a function of frequency $\omega$ for a given momentum $q$, e.g., the cut at $q \ll 2 k_{F}$ of Fig. 1 (at $T=0$ ). The single-pair excitation continuum lies between the $\omega_{-}$and $\omega_{+}$ branches. As known from Refs. 13 and 18, the spectral weight diverges at the lower edge $\omega_{-}$and crosses over smoothly to a multipair continuum at the upper edge $\omega_{+}$.

agrees with Eq. (8), but the nature of the divergency is different according to the order in which the limits $m \rightarrow \infty$ and $\omega \rightarrow v q$ are taken. The fact that these limits do not commute witness the nontrivial role of the irrelevant band-curvature correction. Indeed, the latter cures the TL $\delta$-function singularities [cf. Eq. (8)], providing the exact line shape of the free-fermion DSF [cf. Eq. (7)], and the finite lifetime of the elementary excitations [cf. Eq. (9)]. Moreover, all these results are nonperturbative in band curvature as they require summing over an infinite number of singular terms.

The case of interacting fermions has been the subject of the recent literature. The results are summarized schematically in Fig. 2. The latter shows that the interplay between band curvature and interactions for the DSF is twofold. On the one hand, at high frequencies $(\omega \gg v q)$, this interplay yields a multipair continuum to the DSF. The two-pair continuum was first derived in Ref. 19 and subsequently in Refs. 13 and 21. In Refs. 19 and 21 fermionic techniques were used to derive the two-pair continuum whereas Ref. 13 derived it with the help of the bosonization technique. This high-frequency continuum may be accessed by perturbation theory in curvature and interactions. On the other hand, at low frequencies $(\omega \approx v q)$, a (power-law) divergency of the spectral weight appears at the lower edge $\omega_{-}$and a smooth crossover to the multipair continuum takes place at the upper edge $\omega_{+} \cdot{ }^{13,18}$ Moreover, the width of the single-pair continuum was shown to be affected by interactions ${ }^{13,19}$

$$
\delta \omega \propto \frac{q^{2}}{m^{*}},
$$

where $m^{*}$ gets smaller with increasing repulsive interactions, i.e., the plasmon peak becomes broader. In contrast to the high-frequency part, the low-frequency regime requires a nonperturbative treatment of band curvature. This was already the case for free fermions. Adding interactions of course considerably complicates the problem. This was achieved in Refs. 18 and 19 by fermionic techniques and in Ref. 13 by Bethe ansatz and numerical simulation. As can be seen from Fig. 2 the free-fermion boxlike line shape is there- fore significantly modified by interactions, at both low and high frequencies.

In this frame, our goal will be to calculate the DSF (at small $q$, i.e., the $2 k_{F}$ part is not considered) with the help of the bosonization technique. We will start from a Hubbard chain below half filling and show in detail how the bosonization can be implemented when taking into account bandcurvature corrections to the single-particle spectrum. Our first motivation for using bosonization comes from the fact that it allows us to take forward scattering interactions among the fermions to all orders even though it is essentially perturbative in band curvature. Following the recent Ref. 13, we will reconsider the case of spinless fermions. The exact results available in this case will allow us to discuss the efficiency and limitation of this technique toward a possible resummation of band-curvature corrections. Our second motivation comes from the fact that bosonization allows us to take quite straightforwardly into account the spin 1/2 of the fermions. We will focus on the highly nontrivial coupling between spin and charge degrees of freedom due to band curvature. Within bosonization, this was first noticed in Ref. 15 , which we shall follow (correlation functions were not the main issue of Ref. 15). A spin-charge coupling due to bandspectrum non-linearities was also reported in Ref. 10 within a Bethe ansatz approach to the 1D Hubbard model (correlation functions are not yet computable with this technique). As we will detail below, field and lattice theories agree qualitatively. On this basis, we will explore, with the help of the bosonization technique, the effect of this coupling on the charge and spin density correlation functions of the model. At this point, the reader who is interested more in our results than the derivations may refer directly to Sec. V.

\section{SPINLESS FERMIONS}

\section{A. Model}

In this section, we consider spinless fermions and give some details of the standard bosonization procedure. Starting with free fermions, their Hamiltonian reads

$$
H=\sum_{k} \xi_{k}: c_{k}^{\dagger} c_{k}:
$$

where $c$ and $c^{\dagger}$ are the annihilation and creation operators for the fermions, $\xi_{k}$ is given by Eq. (3), and : : refers to normal ordering. The bosonization procedure is implemented by first focusing on the low-energy sector near the Fermi points $\pm k_{F}$ :

$$
c(x) \sim \psi_{+}(x) e^{-i k_{F} x}+\psi_{-}(x) e^{+i k_{F} x},
$$

where $\psi_{ \pm}$are slow chiral fields. The free-fermion Hamiltonian becomes

$$
\begin{aligned}
H= & i v \int d x\left(: \psi_{+}^{\dagger} \partial_{x} \psi_{+}:-: \psi_{-}^{\dagger} \partial_{x} \psi_{-}:\right) \\
& -\frac{1}{2 m} \int d x\left(: \psi_{+}^{\dagger} \partial_{x x} \psi_{+}:+: \psi_{-}^{\dagger} \partial_{x x} \psi_{-}:\right) .
\end{aligned}
$$

The first term corresponds to the Dirac part related to the linear spectrum. The second term corresponds to the curva- 
ture correction. One may then go to the bosonic representation with the help of the following identity:

$$
\psi_{ \pm}(x)=\frac{1}{\sqrt{2 \pi}}: \exp \left[ \pm i \sqrt{4 \pi} \varphi_{ \pm}(x)\right]:
$$

relating the chiral fermionic fields $\psi_{ \pm}$to the chiral bosonic fields $\varphi_{ \pm}$. The latter may be expressed as a function of the total phase field $\varphi$ and the momentum $\Pi_{\varphi}$ as follows:

$$
\varphi_{ \pm}=\frac{1}{2}\left(\varphi \mp \int_{-\infty}^{x} d x^{\prime} \Pi_{\varphi}\left(x^{\prime}\right)\right) .
$$

The normal ordering of vertex operators is conveniently taken into account with the help of a point-splitting technique. This yields the following operator-product expansions $(\eta= \pm)$ :

$$
\begin{gathered}
\psi_{\eta}^{\dagger}(x) \psi_{\eta}(x)=-\frac{1}{\sqrt{\pi}} \partial_{x} \varphi_{\eta}(x), \\
\psi_{\eta}^{\dagger}(x) \partial_{x} \psi_{\eta}(x)=-i \operatorname{sgn}(\eta):\left[\partial_{x} \varphi_{\eta}(x)\right]^{2}: \\
\psi_{\eta}^{\dagger}(x) \partial_{x x} \psi_{\eta}(x)=\frac{4 \sqrt{\pi}}{3}:\left[\partial_{x} \varphi_{\eta}(x)\right]^{3}:,
\end{gathered}
$$

where the terms that vanish upon integration over space or by symmetry considerations have been neglected. The last line in Eq. (16) displays the nonlinearity of the bosonization arising from band curvature. The fermionic Hamiltonian then takes over the following form:

$$
\begin{aligned}
H= & v \int d x\left[\left(\partial_{x} \varphi_{+}\right)^{2}+\left(\partial_{x} \varphi_{-}\right)^{2}\right] \\
& -\frac{2 \sqrt{\pi}}{3 m} \int d x\left[\left(\partial_{x} \varphi_{+}\right)^{3}+\left(\partial_{x} \varphi_{-}\right)^{3}\right],
\end{aligned}
$$

with the densities

$$
\rho_{+}(x)=-\frac{1}{\sqrt{\pi}} \partial_{x} \varphi_{+}(x), \quad \rho_{-}(x)=-\frac{1}{\sqrt{\pi}} \partial_{x} \varphi_{-}(x) .
$$

The fact that the curvature part is cubic in densities is related to the energy of the Fermi sea for a quadratic spectrum in 1D. ${ }^{22}$ Moreover, such cubic terms lead to a violation of the particle-hole symmetry, $\varphi_{ \pm} \rightarrow-\varphi_{ \pm}$, again a consequence of the nonlinearity of the spectrum away from half filling. Finally, notice that the curvature term in Eq. (17) agrees with the one found in the literature, ${ }^{5,13,16}$ see the Appendix for conventions on notations.

In terms of the total phase and momentum this yields

$$
H=\frac{v}{2} \int d x\left[\Pi_{\varphi}^{2}+\left(\partial_{x} \varphi\right)^{2}\right]-\frac{\sqrt{\pi}}{6 m} \int d x \partial_{x} \varphi\left[3 \Pi_{\varphi}^{2}+\left(\partial_{x} \varphi\right)^{2}\right] .
$$

According to phenomenological bosonization, the added terms $\left(\partial_{x} \varphi\right)^{3}$ and $\partial_{x} \varphi \Pi_{\varphi}^{2}$ are the lowest-order particle-hole symmetry-violating terms compatible with both the U(1) invariance of the Hamiltonian, i.e., $\varphi \rightarrow \varphi+\alpha$ where $\alpha$ gener- ates the translations, and the space-time symmetries of the problem, $\varphi$ is an odd function of $x$ and an even function of $t$ :

$$
\rho=-\frac{1}{\sqrt{\pi}} \partial_{x} \varphi, \quad j=\frac{1}{\sqrt{\pi}} \partial_{t} \varphi,
$$

where $\rho$ is the total density (the smooth part of the density close to $q=0$; the $2 k_{F}$ part has been neglected), $j$ the total current of the fermions, and $\Pi_{\varphi} \sim \partial_{t} \varphi$ [see Eq. (22) below]. Notice also, from Eq. (19), that the original (spinless) fermions correspond to kinks in $\varphi$.

To include forward scattering interactions we follow the usual prescription of rescaling the fields

$$
\Pi_{\varphi} \rightarrow \sqrt{\gamma_{\rho}} \Pi_{\varphi}, \quad \varphi \rightarrow \frac{1}{\sqrt{\gamma_{\rho}}} \varphi, \quad v \rightarrow u_{\rho},
$$

in the Tomonaga-Luttinger part of the Hamiltonian, where

$$
\begin{gathered}
\gamma_{\rho}=\sqrt{\frac{1+y_{4, c} / 2-y_{2, c} / 2}{1+y_{4, c} / 2+y_{2, c} / 2}}, \\
u_{\rho}=v \sqrt{\left(1+y_{4, c} / 2\right)^{2}-\left(y_{2, c} / 2\right)^{2}},
\end{gathered}
$$

and $y_{i, c} \equiv g_{i, c} / \pi v$ is the dimensionless coupling constant in the charge sector. In the following we take $y_{2, c}=y_{4, c}=y_{c}$, which yields

$$
\gamma_{\rho}=\frac{1}{\sqrt{1+y_{c}}}, \quad u_{\rho}=v \sqrt{1+y_{c}} .
$$

The interactions affect the free-fermion Hamiltonian density as

$$
\mathcal{H}=\frac{u_{\rho}}{2}\left(\gamma_{\rho} \Pi_{\varphi}^{2}+\frac{1}{\gamma_{\rho}}\left(\partial_{x} \varphi\right)^{2}\right)-\frac{\sqrt{\pi}}{6 m} \partial_{x} \varphi\left[3 \Pi_{\varphi}^{2}+\left(\partial_{x} \varphi\right)^{2}\right] .
$$

To proceed further, we find it more convenient to go to the Lagrangian representation $\Pi_{\varphi} \rightarrow \partial_{x} \theta$, which yields the following Euclidean action:

$$
\begin{aligned}
S_{E}= & \int d \tau d x\left[\frac{u_{\rho} \gamma_{\rho}}{2}\left(1-\frac{6}{m^{\prime} u_{\rho} \gamma_{\rho}} \partial_{x} \varphi\right)\left(\partial_{x} \theta\right)^{2}-i \partial_{x} \theta \partial_{\tau} \varphi\right. \\
& \left.+\frac{u_{\rho}}{2 \gamma_{\rho}}\left(\partial_{x} \varphi\right)^{2}-\frac{1}{m^{\prime}}\left(\partial_{x} \varphi\right)^{3}\right],
\end{aligned}
$$

where $m^{\prime}=6 m / \sqrt{\pi}$ and $\tau=i t$ is the imaginary time. Integrating over the dual phase field $\theta$ yields

$$
\begin{aligned}
\mathcal{L}_{E}[\varphi]= & \frac{1}{2 \gamma_{\rho} u_{\rho}} \frac{1}{1-\left(6 / m^{\prime} u_{\rho} \gamma_{\rho}\right) \partial_{x} \varphi}\left(\partial_{\tau} \varphi\right)^{2} \\
& +\frac{u_{\rho}}{2 \gamma_{\rho}}\left(\partial_{x} \varphi\right)^{2}-\frac{1}{m^{\prime}}\left(\partial_{x} \varphi\right)^{3} .
\end{aligned}
$$

The nontrivial denominator comes from the conjugated momentum, which reads 


$$
\Pi_{\varphi}=\frac{\partial_{t} \varphi}{u_{\rho} \gamma_{\rho}\left[1-\left(6 / m^{\prime} u_{\rho} \gamma_{\rho}\right) \partial_{x} \varphi\right]}
$$

Expanding the previous expression in the lowest meaningful order in $1 / m$ yields

$$
\begin{aligned}
\mathcal{L}_{E}[\varphi]= & \frac{1}{2 v}\left[\left(\partial_{\tau} \varphi\right)^{2}+u_{\rho}^{2}\left(\partial_{x} \varphi\right)^{2}\right] \\
& +\frac{1}{m^{\prime} v^{2}} \partial_{x} \varphi\left[3\left(\partial_{\tau} \varphi\right)^{2}-v^{2}\left(\partial_{x} \varphi\right)^{2}\right],
\end{aligned}
$$

where we have used the fact that $\gamma_{\rho} u_{\rho}=v$, independent of interactions. $^{23}$

As an alternative to Eq. (23), we may work with the complete action:

$$
\begin{aligned}
\mathcal{L}_{E}[\varphi, \theta]= & \frac{u_{\rho} \gamma_{\rho}}{2}\left(\partial_{x} \theta\right)^{2}+\frac{u_{\rho}}{2 \gamma_{\rho}}\left(\partial_{x} \varphi\right)^{2}-i \partial_{x} \theta \partial_{\tau} \varphi \\
& -\frac{3}{m^{\prime}} \partial_{x} \varphi\left(\partial_{x} \theta\right)^{2}-\frac{1}{m^{\prime}}\left(\partial_{x} \varphi\right)^{3},
\end{aligned}
$$

which does not require any expansion in $1 / \mathrm{m}$.

With the help of Eq. (23) or Eq. (24), we wish to determine the effect of the irrelevant terms appearing due to curvature on the correlation functions of the model. In particular, the long-distance part (close to $q=0$; the $2 k_{F}$ part is neglected) of the polarization operator reads

$$
\Pi(x, \tau)=\frac{2}{\pi}\left\langle\partial_{x} \varphi(x, \tau) \partial_{x} \varphi(0,0)\right\rangle,
$$

where we have taken into account the spin degeneracy. From its expression in Fourier space all we need to compute is the phase correlator $\mathcal{D}$ :

$$
\Pi(i \omega, q)=-\frac{2 q^{2}}{\pi} \mathcal{D}(i \omega, q), \quad \mathcal{D}(i \omega, q)=\left\langle|\varphi(i \omega, q)|^{2}\right\rangle .
$$

Curvature terms make the actions non-Gaussian, a signature of the fact that they induce interactions between the bosonic excitations. We will treat them in perturbation theory in $1 / \mathrm{m}$. Notice that for Eq. (23) curvature generates an infinite number of irrelevant terms, and we have taken into account only the lowest-order terms. This means that our treatment of curvature is essentially perturbative [even if we manage to resum all terms generated by Eq. (23)]. At second order, both models of Eq. (23) and Eq. (24) are equivalent, as will be shown in more detail below.

\section{B. Dynamical structure factor}

We now compute $\mathcal{D}(i \omega, q)$ via bosonization, i.e., in perturbation theory in $1 / \mathrm{m}$. From Eq. (23) the zeroth order reads

$$
\mathcal{D}^{(0)}(i \omega, q)=-\frac{u_{\rho} \gamma_{\rho}}{(i \omega)^{2}-\left(u_{\rho} q\right)^{2}}
$$

from which

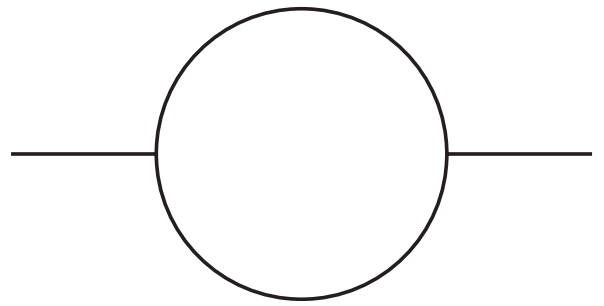

FIG. 3. Second order diagram of the bosonic field theory for the $\varphi$-Lagrangian of Eq. (23) for spinless fermions.

$$
\Pi^{(0)}(i \omega, q)=\frac{2 \gamma_{\rho}}{\pi u_{\rho}} \frac{\left(u_{\rho} q\right)^{2}}{(i \omega)^{2}-\left(u_{\rho} q\right)^{2}},
$$

which agrees with the zero-order term of Eq. (10) in the free-fermion limit: $\gamma_{\rho}=1$ and $u_{\rho}=v$.

In the Euclidean action the cubic perturbation reads

$$
\begin{aligned}
S_{E}= & \frac{i}{m^{\prime} v^{2}} \frac{1}{\beta^{3}} \sum_{\omega_{1}, \omega_{2}, \omega_{3}} \int \frac{d q_{1} d q_{2} d q_{3}}{(2 \pi)^{3}} \delta[1+2+3] \\
& \times\left(3 q_{1} \omega_{2} \omega_{3}-v^{2} q_{1} q_{2} q_{3}\right) \varphi_{\omega_{1}}\left(q_{1}\right) \varphi_{\omega_{2}}\left(q_{2}\right) \varphi_{\omega_{3}}\left(q_{3}\right) .
\end{aligned}
$$

As will be discussed in the following, the knowledge of the self-energy associated with this perturbation (at least in the lowest order) does not imply any meaningful resummation of band-curvature effects at the level of the Dyson equation. We will therefore simply calculate corrections to the bare Bose propagator order by order:

$$
\mathcal{D}(i \omega, q)=\mathcal{D}^{(0)}(i \omega, q)+\mathcal{D}^{(2)}(i \omega, q)+\cdots,
$$

and similarly for the polarization operator. The lowest-order correction is of the second order in band curvature. It reads

$$
\mathcal{D}^{(2)}(i \omega, q)=\mathcal{D}^{(0)}(i \omega, q) \Sigma(i \omega, q) \mathcal{D}^{(0)}(i \omega, q),
$$

where $\Sigma(i \omega, q)$ is the second-order self-energy part and is used without any resummation implied. From the expression of the free bosonic Green function Eq. (26), it will allow us to compute the second-order correction to the polarization operator Eq. (25):

$$
\Pi^{(2)}(i \omega, q)=-\frac{2 \gamma_{\rho}^{2}}{\pi} \frac{\left(u_{\rho} q\right)^{2}}{\left[(i \omega)^{2}-\left(u_{\rho} q\right)^{2}\right]^{2}} \Sigma(i \omega, q) .
$$

For the $\varphi$ Lagrangian the diagrammatic theory assigns a solid line for the bare propagator of Eq. (26) and the second-order self-energy part is displayed in Fig. 3. To compute this selfenergy part one has to be careful in Wick ordering the eightpoint correlation functions generated by the cubic action at second order. This care is due to the fact that, in the $\varphi$ representation, the vertices carry products of frequency and momenta in a nonsymmetric way, which complicates the calculation of the combinatorial factor. The latter is crucial as we will see in the following.

As an alternative to the $\varphi$ representation we may work in the $\varphi, \theta$ representation. As we have noticed in Sec. III A, the $\varphi$ Lagrangian of Eq. (23) is derived by neglecting an infinite number of irrelevant perturbations. On the other hand, no such truncation appears in the $\varphi, \theta$ Lagrangian of Eq. (24). 

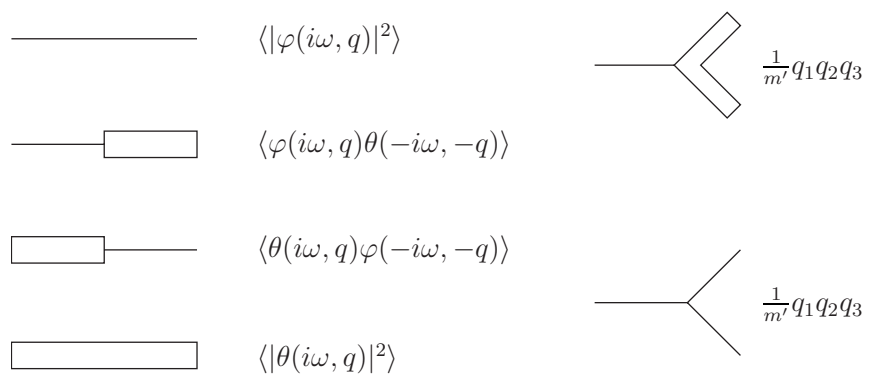

FIG. 4. Quantum field theory associated with the $\varphi, \theta$ Lagrangian of Eq. (24) for spinless fermions.

At the second order of the perturbation theory in curvature, both approaches should yield the same results, while at higher orders the $\varphi, \theta$ Lagrangian has to be used. Moreover, in the $\varphi, \theta$ representation the vertices carry products of momentum in a symmetric way, which simplifies the Wick ordering at the expense of having more diagrams to evaluate. The latter can be seen from the Fourier transform of Eq. (24), which yields the following perturbations:

$$
\begin{aligned}
S_{E}= & -\frac{i}{m^{\prime}} \frac{1}{\beta^{3}} \sum_{\omega_{1}, \omega_{2}, \omega_{3}} \int \frac{d q_{1} d q_{2} d q_{3}}{(2 \pi)^{3}} \delta[1+2+3] \\
& \times q_{1} q_{2} q_{3}\left[3 \varphi_{\omega_{1}}\left(q_{1}\right) \theta_{\omega_{2}}\left(q_{2}\right) \theta_{\omega_{3}}\left(q_{3}\right)\right. \\
& \left.+\varphi_{\omega_{1}}\left(q_{1}\right) \varphi_{\omega_{2}}\left(q_{2}\right) \varphi_{\omega_{3}}\left(q_{3}\right)\right] .
\end{aligned}
$$

The quadratic part of Eq. (24) yields the zero-order correlators

$$
\begin{gathered}
\mathcal{D}_{\varphi \varphi}^{(0)}(i \omega, q)=\left\langle|\varphi(i \omega, q)|^{2}\right\rangle=-\frac{u_{\rho} \gamma_{\rho}}{(i \omega)^{2}-\left(u_{\rho} q\right)^{2}}, \\
\mathcal{D}_{\theta \theta}^{(0)}(i \omega, q)=\left\langle|\theta(i \omega, q)|^{2}\right\rangle=-\frac{u_{\rho}}{\gamma_{\rho}} \frac{1}{(i \omega)^{2}-\left(u_{\rho} q\right)^{2}}, \\
\mathcal{D}_{\varphi \theta}^{(0)}(i \omega, q)=\langle\varphi(i \omega, q) \theta(-i \omega,-q)\rangle=\frac{-i \omega}{q\left[(i \omega)^{2}-\left(u_{\rho} q\right)^{2}\right]}, \\
\mathcal{D}_{\theta \varphi}^{(0)}(i \omega, q)=\mathcal{D}_{\varphi \theta}^{(0)}(i \omega, q) .
\end{gathered}
$$

Notice that, while the charge phase $\varphi$ is related to particlehole pairings, $\mathcal{D}_{\varphi \varphi}^{(0)} \sim \Pi^{(0)}$, its dual $\theta$ is a "superconducting" phase and $\mathcal{D}_{\theta \theta}^{(0)}$ is related to particle-particle pairings. The fact that $\varphi$ and $\theta$ appear on equal footing in Eq. (23) is related to the absence of long-range order (of either the charge-density wave type or the superconducting one) in 1D.

The diagrammatic theory then assigns a solid line for the $\varphi$ field and a double line for the $\theta$ field (see Fig. 4). Expanding the perturbation up to second order and Wick ordering the eight-point correlation functions yields seven different types of diagrams represented in Fig. 5. They are classified according to the number of $\theta$ wings attached to the selfenergy parts. These diagrams, Fig. 5, yield exactly the same self-energy contribution as that of the $\varphi$ representation, Fig. 3.

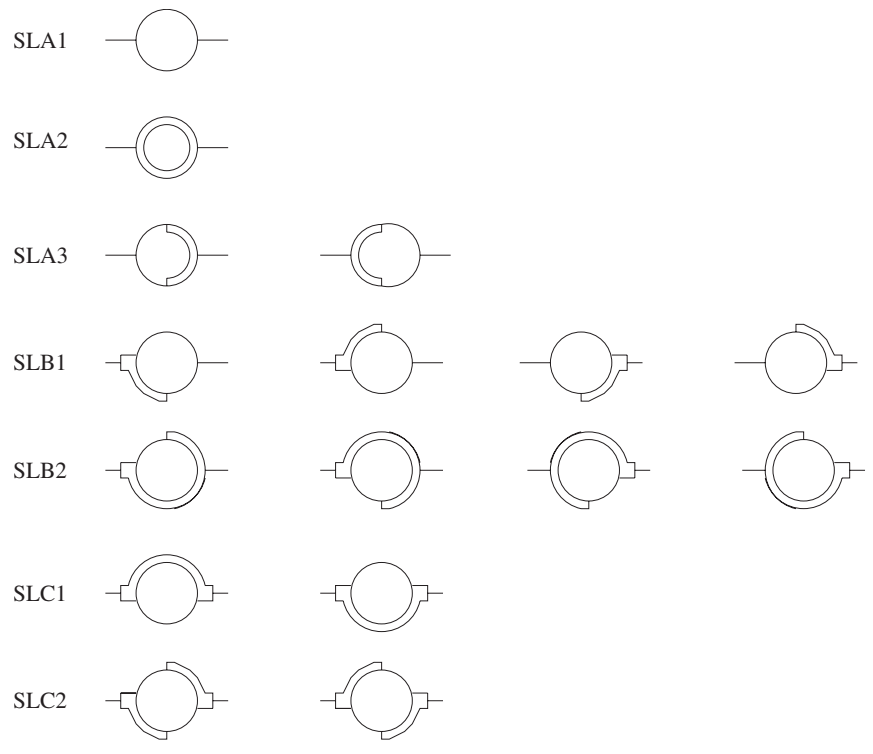

FIG. 5. Second-order diagrams of the bosonic field theory for the $\varphi, \theta$ Lagrangian of Eq. (24) for spinless fermions. SL stands for spinless.

The final result for the small $q$ self-energy reads (at $T$ $=0$ for simplicity)

$$
\begin{aligned}
\operatorname{Im} \Sigma_{\rho}^{R}(\omega, q)= & \frac{\gamma_{\rho}^{2} \pi}{96 u_{\rho}}\left(1+\frac{3}{\gamma_{\rho}^{2}}\right)^{2}\left(\frac{q^{2}}{m}\right)^{2} u_{\rho} q\left(\delta\left[\omega-u_{\rho} q\right]\right. \\
& \left.-\delta\left[\omega+u_{\rho} q\right]\right)+\frac{\gamma_{\rho}^{2} \pi}{64 u_{\rho}}\left(1-\frac{1}{\gamma_{\rho}^{2}}\right)^{2}\left(\frac{q}{m u_{\rho}}\right)^{2} \\
& \times\left[\omega^{2}-\left(u_{\rho} q\right)^{2}\right] \mathcal{F}_{\rho}(\omega, q),
\end{aligned}
$$

where we have restored $m^{\prime}=6 m / \sqrt{\pi}$ and defined the kinematic factor

$$
\mathcal{F}_{\rho}(\omega, q)=\theta\left(\omega-u_{\rho} q\right)-\theta\left(-\omega-u_{\rho} q\right)
$$

\section{Multipair continuum}

Away from the plasmon cone, $\omega \gg u_{\rho}|q|$, Eq. (31) yields the small-wave-vector, high-frequency part of the DSF:

$$
S_{\rho}^{(2)}(\omega, q)=\frac{\left(1-\gamma_{\rho}^{2}\right)^{2}}{32 u_{\rho}}\left(\frac{q^{2}}{m}\right)^{2} \frac{1}{\omega^{2}-\left(u_{\rho} q\right)^{2}} \mathcal{F}_{\rho}(\omega, q)
$$

which is the leading contribution to the DSF in this frequency regime. This tail is by now well known in the literature (cf. Refs. 13, 19, and 21), and describes the decay of a plasmon into two plasmon excitations as can be seen from the vertices of Fig. 4. In fermionic language the plasmon corresponds to a coherent particle-hole pair. As a consequence, the tail is termed the two-pair excitation continuum (see Figs. 1 and 2). Notice that the kinematic factor appearing in Eq. (33) [see also Eq. (32)] is due to the fact that, in 1D, low-energy particle-hole pairs cannot exist for arbitrarily small momentum. 

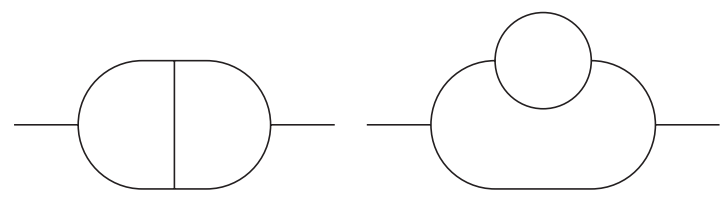

FIG. 6. Fourth-order diagrams of the bosonic field theory for the $\varphi$ Lagrangian of spinless fermions.

\section{Single-pair continuum}

Close to the plasmon cone, $\omega=u_{\rho}|q|$, the total polarization operator may be again conveniently recast in the Matsubara form:

$$
\Pi(i \omega, q)=\Pi^{(0)}(i \omega, q)+\Pi^{(2)}(i \omega, q)+\cdots,
$$

where the ellipsis refers to higher-order band-curvature corrections (cf. the fourth-order contributions of Fig. 6), and from Eqs. (31) and (29)

$$
\Pi^{(2)}(i \omega, q)=\frac{2}{3 \pi u_{\rho}}\left(\frac{3+\gamma_{\rho}^{2}}{4}\right)^{2}\left(\frac{q^{2}}{m}\right)^{2} \frac{\left(u_{\rho} q\right)^{4}}{\left[(i \omega)^{2}-\left(u_{\rho} q\right)^{2}\right]^{3}} .
$$

We now perform a resummation of band-curvature corrections from the second-order self-energy part. For $q \ll k_{F}$, this yields new poles for the dressed polarization operator:

$$
\omega_{ \pm}(q)=u_{\rho}|q| \pm \sqrt{\frac{2}{3}} \frac{3+\gamma_{\rho}^{2}}{4 \sqrt{\gamma_{\rho}}} \frac{q^{2}}{2 m} .
$$

We therefore recover the two-parametric structure of the single-pair continuum. Moreover, up to a numerical prefactor of the order of unity, the width

$$
\delta \omega \propto \frac{q^{2}}{m^{*}}, \quad m^{*}=\frac{4 \sqrt{\gamma_{\rho}}}{3+\gamma_{\rho}^{2}} m,
$$

increases with increasing repulsive interactions. This agrees qualitatively with the exact results of Refs. 13 and 18.

On the other hand, the precise line shape of the DSF in the single-pair continuum (divergency on the lower edge and crossover to the multipair continuum at the upper edge; see Fig. 2) cannot be accessed, even qualitatively, with the help of the second-order self-energy. Indeed, such a partial resummation does not cure the $\delta$-function singularities at the new frequencies of Eq. (36). Higher-order corrections, some of which are represented in Fig. 6, have to be taken into account in order to develop a more efficient resummation scheme. This important issue is, however, beyond the scope of the present paper.

\section{SPINFUL FERMIONS}

\section{A. Model and spin-charge coupling}

Following the previous sections we now move to spinful fermions by introducing an additional internal, spin-1/2, degree of freedom to the fields, $\sigma=\uparrow, \downarrow$, so that

$$
\psi_{ \pm, \sigma}(x)=\frac{1}{\sqrt{2 \pi}}: \exp \left[ \pm i \sqrt{4 \pi} \varphi_{ \pm, \sigma}(x)\right]
$$

$$
\varphi_{ \pm, \sigma}=\frac{1}{2}\left(\varphi_{\sigma} \mp \int_{-\infty}^{x} d x^{\prime} \Pi_{\sigma}\left(x^{\prime}\right)\right) .
$$

This yields

$$
\begin{aligned}
H= & v \sum_{\sigma} \int d x\left[\left(\partial_{x} \varphi_{+, \sigma}\right)^{2}+\left(\partial_{x} \varphi_{-, \sigma}\right)^{2}\right] \\
& -\frac{2 \sqrt{\pi}}{3 m} \int d x\left[\left(\partial_{x} \varphi_{+, \sigma}\right)^{3}+\left(\partial_{x} \varphi_{-, \sigma}\right)^{3}\right],
\end{aligned}
$$

with the densities

$$
\rho_{\eta, \sigma}(x)=-\frac{1}{\sqrt{\pi}} \partial_{x} \varphi_{\eta, \sigma}(x) .
$$

We then introduce the charge and spin fields together with their canonically conjugated fields:

$$
\begin{gathered}
\varphi=\frac{1}{\sqrt{2}}\left(\varphi_{\uparrow}+\varphi_{\downarrow}\right), \quad \sigma=\frac{1}{\sqrt{2}}\left(\varphi_{\uparrow}-\varphi_{\downarrow}\right), \\
\Pi_{\varphi}=\frac{1}{\sqrt{2}}\left(\Pi_{\uparrow}+\Pi_{\downarrow}\right), \quad \Pi_{\sigma}=\frac{1}{\sqrt{2}}\left(\Pi_{\uparrow}-\Pi_{\downarrow}\right) .
\end{gathered}
$$

Following Ref. 15, the Hamiltonian density then takes the following form:

$$
\begin{gathered}
\mathcal{H}=\mathcal{H}_{T L}+\mathcal{H}_{C} \\
\mathcal{H}_{T L}=\frac{u_{\rho}}{2}\left(\gamma_{\rho} \Pi_{\varphi}^{2}+\frac{1}{\gamma_{\rho}}\left(\partial_{x} \varphi\right)^{2}\right)+\frac{u_{\sigma}}{2}\left(\gamma_{\sigma} \Pi_{\sigma}^{2}+\frac{1}{\gamma_{\sigma}}\left(\partial_{x} \sigma\right)^{2}\right) \\
\mathcal{H}_{C}=-\frac{1}{m^{\prime}}\left\{\left(\partial_{x} \varphi\right)^{3}+3 \partial_{x} \varphi \Pi_{\varphi}^{2}+3 \partial_{x} \varphi\left[\left(\partial_{x} \sigma\right)^{2}+\Pi_{\sigma}^{2}\right]\right. \\
\left.+6 \Pi_{\varphi} \partial_{x} \sigma \Pi_{\sigma}\right\}
\end{gathered}
$$

where $m^{\prime}=6 m / \sqrt{\pi}$. In Eqs. (38), interactions in the charge and spin sectors have been included. For the latter we have introduced the following parameters:

$$
\begin{gathered}
\gamma_{\sigma}=\sqrt{\frac{1+y_{4, s} / 2-y_{2, s} / 2}{1+y_{4, s} / 2+y_{2, s} / 2}}, \\
u_{\sigma}=v \sqrt{\left(1+y_{4, s} / 2\right)^{2}-\left(y_{2, s} / 2\right)^{2}},
\end{gathered}
$$

where $y_{i, s} \equiv g_{i, s} / \pi v$ is the dimensionless coupling constant in the spin sector. Just as for the charge sector in the following we take $y_{2, s}=y_{4, s}=y_{s}$, which yields

$$
\gamma_{\sigma}=\frac{1}{\sqrt{1+y_{s}}} \quad(=1), \quad u_{\sigma}=v \sqrt{1+y_{s}} \quad(=v) .
$$

In the absence of curvature $\left(m^{\prime} \rightarrow \infty\right)$ only Eq. (38b) remains, which corresponds to the usual Tomonaga-Luttinger model. Its main feature is the separation of the Hilbert spaces associated with charge and spin sectors the spin and charge parts commute with each other; recall the anomalous commutations $\left[\varphi(x), \Pi_{\varphi}\left(x^{\prime}\right)\right]=i \delta\left(x-x^{\prime}\right)$, and similarly for $\sigma$ and $\Pi_{\sigma}$, 
while other commutators are zero\}. Moreover, as can be seen from

$$
\begin{gathered}
\rho_{\rho}(x)=-\sqrt{\frac{2}{\pi}} \partial_{x} \varphi, \quad \rho_{\sigma}(x)=-\sqrt{\frac{2}{\pi}} \partial_{x} \sigma, \\
j_{\rho}(x)=\sqrt{\frac{2}{\pi}} \partial_{t} \varphi, \quad j_{\sigma}(x)=\sqrt{\frac{2}{\pi}} \partial_{t} \sigma,
\end{gathered}
$$

kinks in $\varphi$ determine the density of fermions, while, independently, kinks in $\sigma$ determine the magnetization density due to the spin of these fermions (we are considering the smooth part of the densities close to $q=0$; the $q=2 k_{F}$ parts are neglected). Charge and spin travel at different velocities $u_{\rho}$ and $u_{\sigma}$, respectively, which are determined by the interactions among the fermions. As a result, charge flies away from spin so that the interacting fermions decompose into two elementary excitations. These are the spinless charged holon and antiholon and the chargeless spin-1/2 spinon (see the textbooks $\left.{ }^{6,7}\right)$. Such elementary excitations do not appear explicitly. Only pairs of these do. They correspond to the physical excitations of the system (see also Refs. 9 and 10). In the spin sector they correspond to spin-singlet and -triplet excitations which are degenerate (they consist of two chargeless spin-1/2 spinons). In the charge sector they correspond to the charge-neutral holon-antiholon excitations otherwise known as particle-hole pairs. The Gaussian nature of the TL model implies that these excitations are free.

In the presence of curvature the physical excitations acquire a finite lifetime. In particular, the first two terms in Eq. (38c) are identical to the ones found in the spinless case. They lead to the decay of the charge excitations (particlehole pairs) within the charge sector. More importantly, the next terms in Eq. (38c) show that spin and charge Hilbert spaces are no longer decoupled when curvature is introduced (see Ref. 15). This opens new channels for the decay of the physical excitations. Indeed, the third and fourth terms couple the charge density to the spin density and current, respectively. The last term describes the coupling between the charge and spin currents. Moreover, we see from Eq. (38c) that spin and charge degrees of freedom do not enter in a symmetric way. As a matter of fact, the decay of spin excitations can only take place by affecting the whole charge density along the wire. These field theory arguments are in qualitative agreement with results obtained from more microscopic approaches. As we know from the exact solution of the Hubbard model (see, e.g., Refs. 9 and 10 for reviews), the spin-charge decoupling exists at the level of the elementary excitations: ${ }^{24}$ holons and spinons. However, below half filling, physical spin excitations involve a rearrangement of charge degrees of freedom, and vice versa. ${ }^{25}$ Only in the low-energy limit, i.e., in the limit of a linear single-particle spectrum, do spin and charge sectors decouple completely.

Even though we have derived Eq. (38) the general form of the Hamiltonian and the particular asymmetry between spin and charge may be understood from general symmetry considerations. This allows a phenomenological approach to the model. As in the spinless case, the fact that all curvature terms are cubic is related to the energy of the Fermi sea for a quadratic single-particle spectrum. It implies that the particle-hole symmetry is violated, which is natural away from half filling for such a spectrum. Moreover, the curvature terms in Eq. (38c) are compatible with the basic symmetries of the problem: $\varphi$ and $\sigma$ are odd functions of $x$ and even functions of $t$ [see Eqs. (40)]. Finally, Eq. (38c) is invariant under $\mathrm{U}(1)$ transformations of the charge fields and $\mathrm{SU}(2)$ transformations of the spin fields. The latter is not quite explicit here since we are using the Abelian bosonization technique. ${ }^{26}$ Nevertheless, as a weak manifestation of the $\mathrm{SU}(2)_{1}$ symmetry, all spin fields in Eq. (38c) are paired, e.g., a term of the form $\partial_{x} \varphi \Pi_{\varphi} \Pi_{\sigma}$ is compatible with the basic symmetries but is not SU(2) invariant. Another consequence of the $\mathrm{SU}(2)$ spin-rotational invariance of the theory (e.g., see Ref. 7) is that $y_{s}=-y_{1} / 2$, where $y_{1}$ is the dimensionless coupling constant arising from backscattering. Thus, from Eq. (39) and in the absence of backscattering, ${ }^{22}$ the spin parameters are the free ones: $u_{\sigma}=v$ and $\gamma_{\sigma}=1$. We will work with general values of these parameters in what follows and set them to their noninteracting values at the end of the calculations.

\section{B. Field theory}

To proceed further, we go to the Lagrangian formulation by introducing the dual fields $\theta_{\rho}$ and $\theta_{\sigma}$, such that $\Pi_{\varphi}=\partial_{x} \theta_{\rho}$ and $\Pi_{\sigma}=\partial_{x} \theta_{\sigma}$. This yields

$$
\begin{aligned}
\mathcal{L}_{E}\left[\varphi, \sigma ; \theta_{\rho}, \theta_{\sigma}\right]= & \frac{u_{\rho} \gamma_{\rho}}{2}\left(\partial_{x} \theta_{\rho}\right)^{2}+\frac{u_{\rho}}{2 \gamma_{\rho}}\left(\partial_{x} \varphi\right)^{2}-i \partial_{x} \theta_{\rho} \partial_{\tau} \varphi \\
& +\frac{u_{\sigma} \gamma_{\sigma}}{2}\left(\partial_{x} \theta_{\sigma}\right)^{2}+\frac{u_{\sigma}}{2 \gamma_{\sigma}}\left(\partial_{x} \sigma\right)^{2}-i \partial_{x} \theta_{\sigma} \partial_{\tau} \sigma \\
& -\frac{1}{m^{\prime}} \partial_{x} \varphi\left[\left(\partial_{x} \varphi\right)^{2}+3\left(\partial_{x} \theta_{\rho}\right)^{2}\right] \\
& -\frac{3}{m^{\prime}} \partial_{x} \varphi\left[\left(\partial_{x} \sigma\right)^{2}+\left(\partial_{x} \theta_{\sigma}\right)^{2}\right] \\
& -\frac{6}{m^{\prime}} \partial_{x} \theta_{\rho} \partial_{x} \sigma \partial_{x} \theta_{\sigma} .
\end{aligned}
$$

The field theory associated with Eq. (41) is defined in Fig. 7. In the charge sector, bare correlators are given by Eqs. (30) with $\theta \equiv \theta_{\rho}$. Following the spinless case, the correlator $\mathcal{D}_{\varphi \varphi}^{(0)}$ is associated with the particle-hole pair excitations. These are charge-neutral excitations, even though we will often refer to them as charge excitations, for simplicity, as holons and antiholons never appear explicitly. Moreover, as there is no spin-charge coupling at the level of the bare propagators, these charge-neutral excitations are spinless. In the spin sector, the bare correlators read

$$
\begin{gathered}
\mathcal{D}_{\sigma \sigma}^{(0)}(i \omega, q)=\left\langle|\sigma(i \omega, q)|^{2}\right\rangle=-\frac{u_{\sigma} \gamma_{\sigma}}{(i \omega)^{2}-\left(u_{\sigma} q\right)^{2}}, \\
\mathcal{D}_{\theta_{\sigma} \theta_{\sigma}}^{(0)}(i \omega, q)=\left\langle\left|\theta_{\sigma}(i \omega, q)\right|^{2}\right\rangle=-\frac{u_{\sigma}}{\gamma_{\sigma}} \frac{1}{(i \omega)^{2}-\left(u_{\sigma} q\right)^{2}},
\end{gathered}
$$




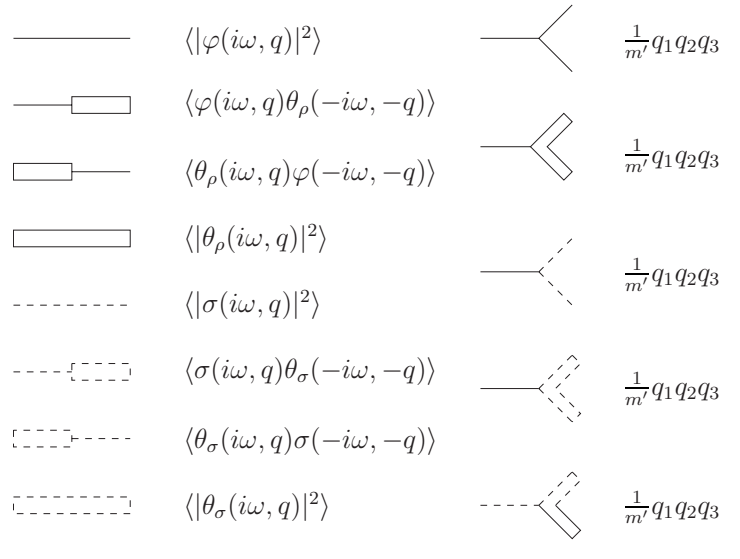

FIG. 7. Quantum field theory associated with the Lagrangian of Eq. (41) for spinful fermions.

$$
\begin{gathered}
\mathcal{D}_{\sigma \theta_{\sigma}}^{(0)}(i \omega, q)=\left\langle\sigma(i \omega, q) \theta_{\sigma}(-i \omega,-q)\right\rangle=\frac{-i \omega}{q\left[(i \omega)^{2}-\left(u_{\sigma} q\right)^{2}\right]}, \\
\mathcal{D}_{\theta_{\sigma} \sigma}^{(0)}(i \omega, q)=\mathcal{D}_{\sigma \theta_{\sigma}}^{(0)}(i \omega, q),
\end{gathered}
$$

where $\mathcal{D}_{\sigma \sigma}^{(0)}$ is associated with spin-singlet or -triplet excitations. We shall often refer to them as spin excitations, for simplicity, as spinons never appear explicitly. They are chargeless.

Alternatively, we may also integrate over the dual fields in Eq. (41) and work with the following reduced Lagrangian:

$$
\begin{aligned}
\mathcal{L}_{E}[\varphi, \sigma]= & \frac{1}{2 \gamma_{\rho}}\left(\frac{1}{u_{\rho}}\left(\partial_{\tau} \varphi\right)^{2}+u_{\rho}\left(\partial_{x} \varphi\right)^{2}\right) \\
& +\frac{1}{2 \gamma_{\sigma}}\left(\frac{1}{u_{\sigma}}\left(\partial_{\tau} \sigma\right)^{2}+u_{\sigma}\left(\partial_{x} \sigma\right)^{2}\right) \\
& +\frac{1}{m^{\prime} v^{2}} \partial_{x} \varphi\left[3\left(\partial_{\tau} \varphi\right)^{2}-v^{2}\left(\partial_{x} \varphi\right)^{2}\right] \\
& +\frac{3}{m^{\prime} v^{2}} \partial_{x} \varphi\left[\left(\partial_{\tau} \sigma\right)^{2}-v^{2}\left(\partial_{x} \sigma\right)^{2}\right]+\frac{6}{m^{\prime} v^{2}} \partial_{\tau} \varphi \partial_{x} \sigma \partial_{\tau} \sigma
\end{aligned}
$$

where only the lowest-order terms in $1 / \mathrm{m}^{\prime}$ have been taken into account. The corresponding field theory is given in Fig. 8.

With the help of the models of Eqs. (41) and (43), we will determine the effects of the spin-charge mixing terms arising from band curvature on the response functions of the system

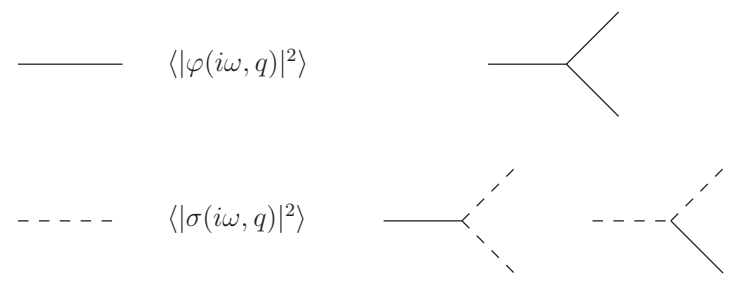

FIG. 8. Quantum field theory related to the Lagrangian of Eq. (43) for spinful fermions.
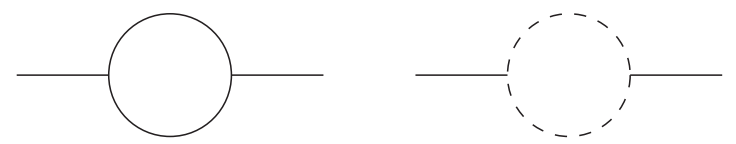

FIG. 9. Second-order diagrams contributing to the charge density correlation function generated from the $\varphi, \sigma$ Lagrangian of Eq. (43) for spinful fermions.

at small wave vector $q$. There are two of them: the charge DSF and the spin (or magnetic) DSF [mixed correlators of the type $\langle\varphi(i \omega, q) \sigma(-i \omega,-q)\rangle$ are zero within the present field theory because there is no spin-charge mixing at the level of the bare propagators].

\section{Dynamical charge structure factor}

We first compute the second-order corrections in band curvature to the charge DSF of spinful fermions. As for the case of spinless fermions, it corresponds to the imaginary part of the related correction for the polarization operator:

$$
\Pi_{\rho}^{(2)}(i \omega, q)=-\frac{2 \gamma_{\rho}^{2}}{\pi} \frac{\left(u_{\rho} q\right)^{2}}{\left[(i \omega)^{2}-\left(u_{\rho} q\right)^{2}\right]^{2}} \Sigma_{\rho}(i \omega, q),
$$

where the self-energy part corresponds to the diagrams of Fig. 9 in the $\varphi, \sigma$ representation. The latter include a contribution that is similar to the spinless case: the first diagram in Fig. 9, arising from the decay of a charge(-neutral) boson into two charge(-neutral) bosons (see the first vertex in Fig. 8 ). Because of band curvature, spin-charge separation is violated and spin degrees of freedom provide another channel for the decay of the charge boson. This channel corresponds to the second vertex of Fig. 8 whereby the charge boson decays into two spin bosons (each corresponding to a spinsinglet excitation). The corresponding diagram, second-order in band curvature, is the second diagram of Fig. 9.

In the $\varphi, \sigma, \theta_{\rho}, \theta_{\sigma}$ representation of Eq. (41) the new diagram is given by the diagrams of Fig. 10 where the same classification as in the spinless case Fig. 5 has been used, i.e., diagrams are classified according to the number of $\theta$ wings attached to their self-energy parts. The increased number of diagrams in Fig. 10 with respect to Fig. 9 reflects the nontrivial nature of the vertices of the cubic field theory under consideration.

The diagrams of Figs. 9 and 10 have the same structure as those of the corresponding spinless case, Figs. 3 and 5, respectively. In the case where charge and spin parameters are equal $\left(\gamma_{\rho}=\gamma_{\sigma}=\gamma\right.$ and $\left.u_{\rho}=u_{\sigma}=u\right)$, the free-fermion case being a special subcase $(\gamma=1$ and $u=v)$, the diagrams of Fig. 9 reduce to the one of Fig. 3 with an additional factor of 2, the spin degeneracy. Similarly, the diagrams of Fig. 10 add with those of Fig. 5 and yield an overall factor of 2. To appreciate the spin-charge coupling one has therefore to impose an inequivalence between these degrees of freedom.

This arises naturally for arbitrary values of the parameters, $\gamma_{\rho} \neq \gamma_{\sigma}$. The total self-energy, close to $q=0$, of interacting spinful fermions then reads (at $T=0$ ) 


$$
\begin{aligned}
\operatorname{Im} \Sigma_{\rho}^{R}(\omega, q)= & \frac{\pi}{96}\left(\frac{q^{2}}{m}\right)^{2}\left[\frac{\gamma_{\rho}^{2}}{u_{\rho}}\left(1+\frac{3}{\gamma_{\rho}^{2}}\right)^{2} u_{\rho} q\left(\delta\left[\omega-u_{\rho} q\right]-\delta\left[\omega+u_{\rho} q\right)\right)+\frac{\gamma_{\sigma}^{2}}{u_{\sigma}}\left(1+\frac{1}{\gamma_{\sigma}^{2}}+\frac{2}{\gamma_{\sigma} \gamma_{\rho}}\right)^{2} u_{\sigma} q\left(\delta\left[\omega-u_{\sigma} q\right]-\delta\left[\omega+u_{\sigma} q\right]\right)\right] \\
& +\frac{\gamma_{\rho}^{2} \pi}{64 u_{\rho}}\left(1-\frac{1}{\gamma_{\rho}^{2}}\right)^{2}\left(\frac{q}{m u_{\rho}}\right)^{2}\left[\omega^{2}-\left(u_{\rho} q\right)^{2}\right] \mathcal{F}_{\rho}(\omega, q)+\frac{\gamma_{\sigma}^{2} \pi}{64 u_{\sigma}}\left(1-\frac{1}{\gamma_{\sigma}^{2}}\right)^{2}\left(\frac{q}{m u_{\sigma}}\right)^{2}\left[\omega^{2}-\left(u_{\sigma} q\right)^{2}\right] \mathcal{F}_{\sigma}(\omega, q),
\end{aligned}
$$

where the kinematic factor reads

$$
\mathcal{F}_{\sigma}(\omega, q)=\theta\left(\omega-u_{\sigma} q\right)-\theta\left(-\omega-u_{\sigma} q\right)
$$

This general result shows that the density correlation function of spinful fermions has two peaks: a charge peak at $\omega$ $=u_{\rho} q$ but also a spin peak at $\omega=u_{\sigma} q$, so that part of the charge spectral weight is carried by the spin singlet. The additional spin peak to the DSF is a witness of the spincharge coupling due to band curvature. It arises from the opening of a new channel for the decay of charge(-neutral) excitations, i.e., into two gapless spin singlets traveling at $u_{\sigma}<u_{\rho}$. The sharpness of this spin peak translates the coherence of the rearrangement of the background charge due to the spin singlet.

In the absence of backscattering, i.e., for $\gamma_{\sigma}=1$ and $u_{\sigma}$ $=v$, Eq. (45) simplifies as

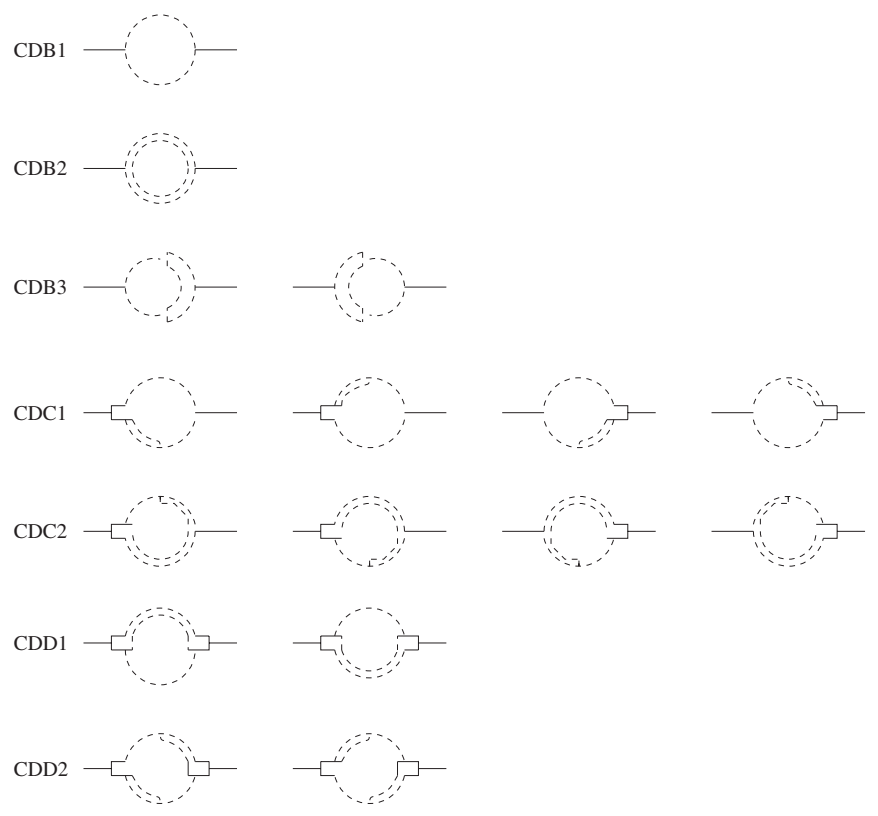

FIG. 10. Second-order diagrams mixing spin and charge degrees of freedom and contributing to the charge density correlation function of spinful fermions. These diagrams are generated with the $\varphi, \sigma, \theta_{\rho}, \theta_{\sigma}$ Lagrangian of Eq. (41). The self-energy parts depend only on the spin degrees of freedom. CD stands for charge density. The set CDA is identical to the diagrams appearing in the spinless case (Fig. 5) and is not displayed. Notice that, up to the fact that the self-energy depends only on the spin degrees of freedom, these diagrams are the same as those of the spinless case, Fig. 5.

$$
\begin{aligned}
\operatorname{Im} \Sigma_{\rho}^{R}(\omega, q)= & \frac{\gamma_{\rho}^{2} \pi}{96 u_{\rho}}\left(1+\frac{3}{\gamma_{\rho}^{2}}\right)^{2}\left(\frac{q^{2}}{m}\right)^{2} \\
& \times u_{\rho} q\left(\delta\left[\omega-u_{\rho} q\right]-\delta\left[\omega+u_{\rho} q\right]\right) \\
& +\frac{\pi}{24 v}\left(1+\frac{1}{\gamma_{\rho}}\right)^{2}\left(\frac{q^{2}}{m}\right)^{2} \\
& \times v q(\delta[\omega-v q]-\delta[\omega+v q]) \\
& +\frac{\gamma_{\rho}^{2} \pi}{64 u_{\rho}}\left(1-\frac{1}{\gamma_{\rho}^{2}}\right)^{2}\left(\frac{q}{m u_{\rho}}\right)^{2} \\
& \times\left[\omega^{2}-\left(u_{\rho} q\right)^{2}\right] \mathcal{F}_{\rho}(\omega, q) .
\end{aligned}
$$

\section{Multipair continuum}

Equation (45) shows that both charge and spin peaks have tails due to the interactions between the fermions composing the bosonic excitations. For $\omega \gg u_{\rho}|q|$ and from Eqs. (45) and (44), the leading contribution to the charge DSF reads

$$
\begin{aligned}
S_{\rho}^{(2)}(\omega, q)= & \frac{\left(1-\gamma_{\rho}^{2}\right)^{2}}{32 u_{\rho}}\left(\frac{q^{2}}{m}\right)^{2} \frac{1}{\omega^{2}-\left(u_{\rho} q\right)^{2}} \mathcal{F}_{\rho}(\omega, q) \\
& +\frac{\left(1-\gamma_{\sigma}^{2}\right)^{2}}{32 u_{\sigma}}\left(\frac{q^{2}}{m}\right)^{2}\left(\frac{u_{\rho} \gamma_{\rho}}{u_{\sigma} \gamma_{\sigma}}\right)^{2} \frac{\omega^{2}-\left(u_{\sigma} q\right)^{2}}{\left[\omega^{2}-\left(u_{\rho} q\right)^{2}\right]^{2}} \mathcal{F}_{\sigma}(\omega, q) .
\end{aligned}
$$

Notice that, in the frequency range $u_{\sigma}|q| \ll \omega \ll u_{\rho}|q|$, a small spectral weight is provided by the spin excitations.

In the limit where backscattering is neglected, Eq. (47), the spin peak at $\omega=v|q|$ has no long-range tails and for strong repulsive interactions, $\gamma_{\rho} \ll 1$, is well separated from the charge peak at $\omega=u_{\rho}|q|=v|q| / \gamma_{\rho} \gg v|q|$.

\section{Single-pair continuum}

We now focus on the vicinity of the charge peak, $\omega$ $\approx u_{\rho}|q|$ and neglect backscattering, so that charge and spin peaks are well separated from each other. Taking into account the leading contribution Eq. (27), the total polarization operator up to second order in band curvature reads

$$
\Pi(i \omega, q)=\Pi^{(0)}(i \omega, q)+\Pi^{(2)}(i \omega, q)+\cdots,
$$

where the ellipsis refers to higher-order corrections, and, from Eqs. (47) and (44), the second-order correction to the polarization operator reads 

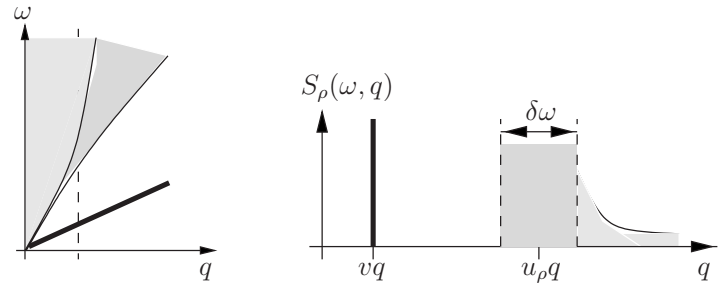

FIG. 11. Schematic view on the low-momentum $\left(q \ll k_{F}\right)$ part of the spectrum of excitation of spinful fermions and the corresponding charge DSF. Interactions are present in the charge sector $\left(\gamma_{\rho}\right.$ $<1)$ while the spin sector is free $\left(\gamma_{\sigma}=1\right)$. Because of the violation of spin-charge separation due to band curvature two peaks appear. The higher one corresponds to the usual charge peak and has a high-frequency tail (displayed in light gray). The lower one is the spin peak. Within bosonization, the width of the spin peak and the precise line shape of the DSF are not accessible.

$$
\begin{aligned}
\Pi^{(2)}(i \omega, q)= & \frac{\gamma_{\rho}^{2}}{6 \pi}\left(\frac{q^{2}}{m}\right)^{2}\left[\frac{\gamma_{\rho}^{2}}{4 u_{\rho}}\left(1+\frac{3}{\gamma_{\rho}^{2}}\right)^{2} \frac{\left(u_{\rho} q\right)^{4}}{\left[(i \omega)^{2}-\left(u_{\rho} q\right)^{2}\right]^{3}}\right. \\
& \left.+\frac{1}{v}\left(1+\frac{1}{\gamma_{\rho}}\right)^{2} \frac{(v q)^{2}\left(u_{\rho} q\right)^{2}}{\left[(i \omega)^{2}-(v q)^{2}\right]\left[(i \omega)^{2}-\left(u_{\rho} q\right)^{2}\right]^{2}}\right] .
\end{aligned}
$$

In Eq. (50) the second term arises from the spin-charge coupling. If we perform a re-summation of the second-order results, the polarization operator acquires new poles at the frequencies solving

$$
\begin{aligned}
{\left[\omega^{2}-\right.} & \left.\left(u_{\rho} q\right)^{2}\right]^{2}\left[\omega^{2}-(v q)^{2}\right] \\
= & \frac{2}{3 \gamma_{\rho}}\left(\frac{3+\gamma^{2}}{4}\right)^{2}\left(\frac{q^{2}}{m}\right)^{2}\left(u_{\rho} q\right)^{2}\left[\omega^{2}-(v q)^{2}\right] \\
& +\frac{2}{3 \gamma_{\rho}}\left(\frac{1+\gamma}{2}\right)^{2}\left(\frac{q^{2}}{m}\right)^{2}(v q)^{2}\left[\omega^{2}-\left(u_{\rho} q\right)^{2}\right] .
\end{aligned}
$$

This equation is second order in $\omega^{2}-\left(u_{\rho} q\right)^{2}$ from which we recover the two-parametric nature of the charge peak. For $q / k_{F} \ll 1$, the latter reads

$$
\omega(q) \approx u_{\rho}^{*}|q| \pm \frac{q^{2}}{2 m^{*}}+O\left(\left(q / k_{F}\right)^{3}\right),
$$

where $m^{*}$ is still given by Eq. (37). At this order the presence of the spin peak does not affect the width of the charge peak. The main effect of the spin-charge coupling, within the present resummation based on the second-order self-energy part, is then to shift the velocity of the charge excitations, $u_{\rho}^{*} \approx u_{\rho}\left[1-\left(q / k_{F}\right)^{2}\right]$.

On the other hand, Eq. (51) is first order in $\omega^{2}-(v q)^{2}$. This implies that, within a resummation based on the secondorder self-energy part, the spin peak remains single parametric with a velocity slightly renormalized by the band curvature, $v^{*} \approx v\left[1-\left(q / k_{F}\right)^{2}\right]$.

Our results are schematically displayed in Fig. 11. Within the present, perturbative approach we cannot access the precise line shape of the charge DSF in the vicinity of $\omega$ $\approx u_{\rho}|q|$ and $\omega \approx v|q|$.
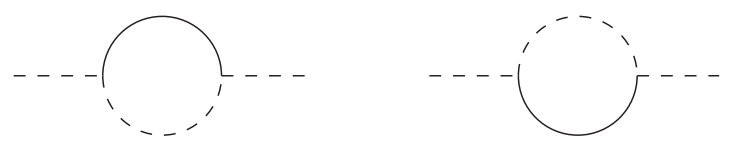

FIG. 12. Second-order diagrams contributing to the spin-density correlation function and generated from the $\varphi, \sigma$ Lagrangian of Eq. (43) for spinful fermions.

\section{Dynamical spin structure factor}

We now focus on the spin degrees of freedom. The longdistance part (close to $q=0$; the $2 k_{F}$ part is neglected) of the spin-density correlation function is defined as

$$
\chi(x, \tau)=\frac{2}{\pi}\left\langle\partial_{x} \sigma(x, \tau) \partial_{x} \sigma(0,0)\right\rangle,
$$

and, in Fourier space, reads

$$
\chi(i \omega, q)=-\frac{q^{2}}{\pi} \mathcal{D}_{\sigma \sigma}(i \omega, q) .
$$

The dynamic spin structure factor corresponds to the dissipative part of this susceptibility,

$$
S_{\sigma}(\omega, q)=-\operatorname{Im} \chi^{R}(\omega, q) .
$$

In the absence of curvature the result is well known:

$$
S_{\sigma}^{0}(\omega, q)=\frac{\gamma_{\sigma}}{u_{\sigma}}\left(u_{\sigma} q\right)^{2} \delta\left[\omega-u_{\sigma}|q|\right]
$$

with obvious similarity to the dynamical charge structure factor.

Including curvature, the second-order correction to the spin density correlation function reads

$$
\chi^{(2)}(i \omega, q)=-\frac{2 \gamma_{\sigma}^{2}}{\pi} \frac{\left(u_{\sigma} q\right)^{2}}{\left[(i \omega)^{2}-\left(u_{\sigma} q\right)^{2}\right]^{2}} \Sigma_{\sigma}(i \omega, q),
$$

where the self-energy $\Sigma_{\sigma}$ is given by the diagrams of Fig. 12 in the the $\varphi, \sigma$ representation of the Lagrangian Eq. (43). These diagrams are built from the third vertex displayed in Fig. 8 which shows that a spin excitation (singlet, triplet) can decay only into a mixed spin (singlet, triplet)-charge-neutral excitation. In contrast to charge fluctuations which had two channels through which they could decay (one of them not affecting the spin degrees of freedom and therefore surviving in the spinless case) the decay of spin fluctuations cannot proceed without affecting the whole charge background.

In the $\varphi, \sigma, \theta_{\rho}, \theta_{\sigma}$ representation of the Lagrangian Eq. (41), which reveals the nontrivial nature of the vertices of the cubic field theory, these mixed spin-charge self-energy parts are displayed on Fig. 13.

In the case where spin and charge degrees of freedom are equivalent $\left(\gamma_{\rho}=\gamma_{\sigma}=\gamma\right.$ and $\left.u_{\rho}=u_{\sigma}=u\right)$, the free-fermion case being a special subcase $(\gamma=1$ and $u=v)$, spin and charge bare propagators are equal so that there is no more difference between the solid and dashed lines in Figs. 12 and 13. One can then easily check that in this case the diagrams of Figs. 12 and 13 are identical to those of Figs. 3 and 5, respectively, with an additional factor of 2 due to the spin degeneracy. 
All nontrivial effects that we shall observe below are therefore related to the fact that spin and charge excitations travel at different velocities $\left(\gamma_{\rho} \neq \gamma_{\sigma}\right)$. Even though calculations may be carried out for arbitrary spin and charge param- eters we will focus here, for simplicity, on the case where backscattering is neglected, so that the spin sector is free ( $\gamma_{\sigma}=1$ and $u_{\sigma}=v$ ). The self-energy then reads (at $T=0$ for simplicity)

$$
\begin{aligned}
\operatorname{Im} \Sigma_{\sigma}^{R}(\omega, q)= & \frac{\gamma_{\rho} \pi}{8 v}\left(1+\frac{1}{\gamma_{\rho}}\right)^{2}\left(1+\frac{\omega}{v q}\right)^{2}\left(\frac{q^{2}}{m}\right)^{2} v q \frac{(\omega-v q)\left(\omega-u_{\rho} q\right)}{\left|u_{\rho}-v\right|^{3} q^{3}}\left[\theta\left(-\frac{u_{\rho}(\omega-v q)}{u_{\rho}-v}\right)-\theta\left(-\frac{v\left(\omega-u_{\rho} q\right)}{u_{\rho}-v}\right)\right] \\
& +\frac{\gamma_{\rho} \pi}{8 v}\left(1+\frac{1}{\gamma_{\rho}}\right)^{2}\left(1-\frac{\omega}{v q}\right)^{2}\left(\frac{q^{2}}{m}\right)^{2} v q \frac{(\omega+v q)\left(\omega+u_{\rho} q\right)}{\left|u_{\rho}-v\right|^{3} q^{3}}\left[\theta\left(-\frac{u_{\rho}(\omega+v q)}{u_{\rho}-v}\right)-\theta\left(-\frac{v\left(\omega+u_{\rho} q\right)}{u_{\rho}-v}\right)\right] \\
& +\frac{\gamma_{\rho} \pi}{8}\left(1-\frac{1}{\gamma_{\rho}}\right)^{2}\left(1-\frac{\omega}{v q}\right)^{2}\left(\frac{q}{m}\right)^{2} \frac{1}{\left|u_{\rho}+v\right|^{3}}(\omega+v q)\left(\omega-u_{\rho} q\right)\left[\theta\left(\frac{v\left(\omega-u_{\rho} q\right)}{u_{\rho}+v}\right)-\theta\left(-\frac{u_{\rho}(\omega+v q)}{u_{\rho}+v}\right)\right] \\
& +\frac{\gamma_{\rho} \pi}{8}\left(1-\frac{1}{\gamma_{\rho}}\right)^{2}\left(1+\frac{\omega}{v q}\right)^{2}\left(\frac{q}{m}\right)^{2} \frac{1}{\left|u_{\rho}+v\right|^{3}}(\omega-v q)\left(\omega+u_{\rho} q\right)\left[\theta\left(\frac{v\left(\omega+u_{\rho} q\right)}{u_{\rho}+v}\right)-\theta\left(-\frac{u_{\rho}(\omega-v q)}{u_{\rho}+v}\right)\right] .
\end{aligned}
$$

Notice that in the limit where $\gamma_{\rho} \rightarrow 1$ in Eq. (58) the first two terms become $\delta$ functions centered around $\pm v q$ and the last two terms vanish. On the other hand, for $\gamma_{\rho}<1$, the combined effect of repulsive interactions and second-order curvature effects broadens the peaks at $\pm v q$ by transferring spectral weight to frequencies reaching the charge frequency $\left(u_{\rho} q\right)$ with additional long-range tails. This is more conveniently seen on the magnetic DSF. The latter is nonzero all the way between the spin and charge frequencies:

$$
\begin{aligned}
S_{\sigma}^{(2)}(\omega, q)= & \frac{\gamma_{\rho}}{4 v}\left(\frac{q}{m v}\right)^{2}\left[\theta\left(-\omega+u_{\rho} q\right)-\theta(-\omega+v q)\right] \\
& \times\left(\frac{\left(1 / \gamma_{\rho}+1\right)^{2}}{\left|1 / \gamma_{\rho}-1\right|^{3}} \frac{\omega-u_{\rho} q}{\omega-v q}+\frac{\left(1 / \gamma_{\rho}-1\right)^{2}}{\left|1 / \gamma_{\rho}+1\right|^{3}} \frac{\omega+u_{\rho} q}{\omega-v q}\right),
\end{aligned}
$$

where, for simplicity, we have set $q>0$ and focused on $\omega$ $>v q$.

At $\omega=v q$ this expression diverges but only algebraically and not as a $\delta$ function as for the zero-order term or in the absence of interactions.

At $\omega=u_{\rho} q$ the magnetic DSF is finite and equals

$$
S_{\sigma}^{(2)}\left(u_{\rho} q, q\right)=\frac{1}{2 v} \frac{1 / \gamma_{\rho}-1}{\left|1 / \gamma_{\rho}+1\right|^{3}}\left(\frac{q}{m v}\right)^{2},
$$

so that part of the magnetic spectral weight is carried by the charge excitations.

At large frequencies $\omega \gg u_{\rho} q$, tails appear and read

$$
\begin{aligned}
S_{\sigma}^{(2)}(\omega, q)= & \frac{\gamma_{\rho}}{2 v}\left(\frac{1 / \gamma_{\rho}-1}{1 / \gamma_{\rho}+1}\right)^{2}\left(\frac{q^{2}}{m}\right)^{2} \frac{\theta\left(\omega-u_{\rho} q\right)}{\omega^{2}-(v q)^{2}} \\
& +\frac{\gamma_{\rho}}{2 v} \frac{\left(1 / \gamma_{\rho}-1\right)^{2}}{\left|1 / \gamma_{\rho}+1\right|^{3}}\left(\frac{q}{m v}\right)^{2} \theta\left(\omega-u_{\rho} q\right) .
\end{aligned}
$$

Notice that the second term in Eq. (61) yields a nonzero dissipation at infinite frequencies. Nevertheless, the spinstructure factor satisfies the sum rule

$$
\int_{0}^{\infty} d \omega \omega S_{\sigma}(\omega, q)=\frac{v^{*} q^{2}}{2},
$$

but with a renormalized spin velocity $v^{*}$, which reads

$$
\frac{v^{*}}{v}=1+\frac{\gamma_{\rho}^{2}}{4} \frac{\left(1-\gamma_{\rho}\right)^{2}}{\left|1+\gamma_{\rho}\right|^{3}}, \quad v<v^{*}<u_{\rho} .
$$

In Eq. (62) a large-frequency cutoff at $\epsilon_{F}=m v^{2}$ has been included, and the final result was obtained after sending this cutoff to infinity. The renormalization of the spin velocity by interactions in the charge sector is precisely due to the last term in Eq. (61). It is a signature that the physical excitations carrying the magnetic spectral weight, in the tails of the spinstructure factor, are spin (-singlet or -triplet) excitations dressed by charge-neutral ones.

We therefore see that band curvature softens the singularities of the spin structure factor already at the second order of perturbation theory. This effect arises from spin-charge mixing and is more dramatic than for the charge DSF. The latter was also affected by the violation of the spin-charge separation due to band curvature[see the double-peak structure in Eqs. (47) and (50) and Fig. 11] but its low-frequency part was still $\delta$ singular at second order in band curvature. The sharpness of both charge and spin peaks in the charge DSF was due to the decay of charge fluctuations into either a pair of charge-neutral excitations or a pair of spin-singlet excitations, both coherently traveling at velocities $u_{\rho}$ and $u_{\sigma}$, respectively. On the other hand spin fluctuations decay via spin(-singlet or -triplet)-charge(-neutral) excitations. Both components of this pair lead to rearrangement of the background charge along the wire. ${ }^{27}$ But they travel at different velocities. As a consequence, the redistribution of magnetic spectral weight is incoherent and affects an infinite number 


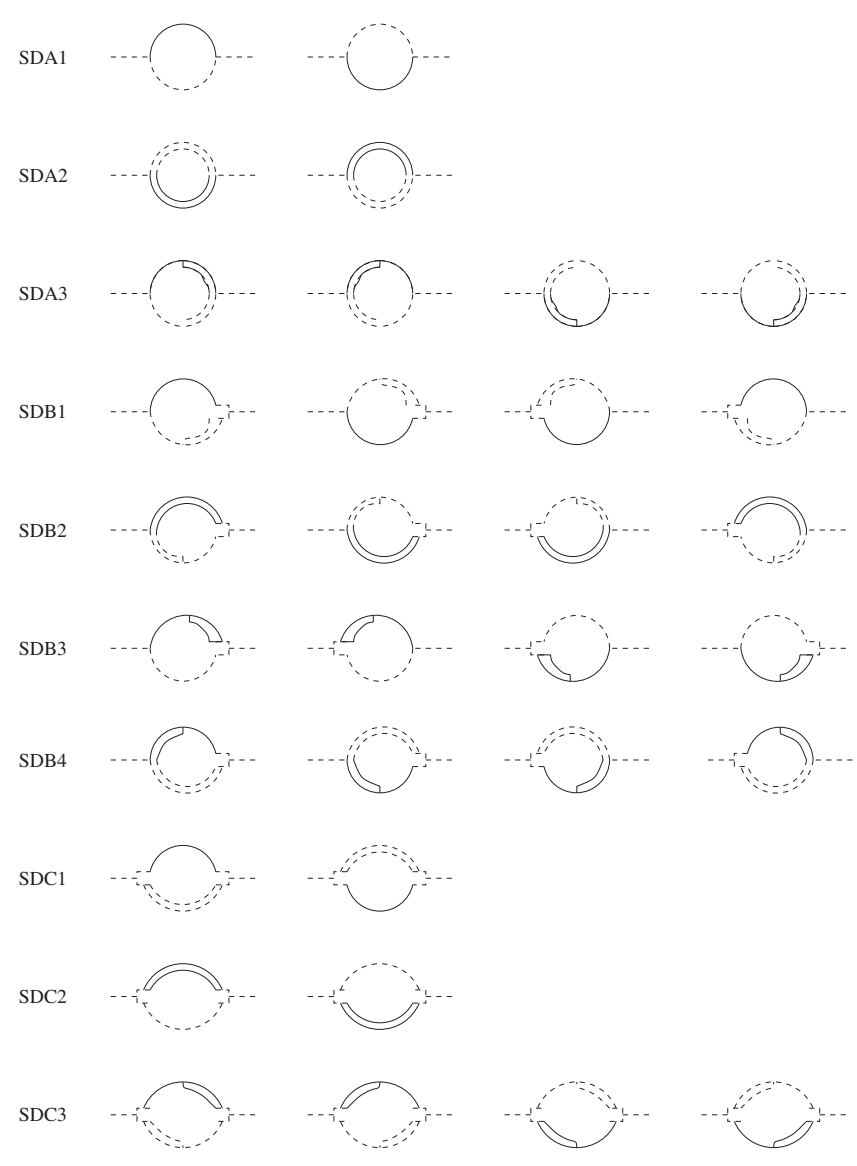

FIG. 13. Second-order diagrams contributing to the spin-density correlation function and generated from the $\varphi, \sigma, \theta_{\rho}, \theta_{\sigma}$ Lagrangian of Eq. (41) for spinful fermions. All the self-energy parts mix spin and charge. SD stands for spin density. In the limit where spin and charge degrees of freedom are equal, these diagrams reduce to those of Fig. 5 up to spin degeneracy.

of modes. The magnetic DSF is plotted in Fig. 14 for different values of the interactions in the charge sector. Because of the spin-charge coupling just described more spectral weight is transferred from the spin peak at $\omega=v q$ to the charge peak at $\omega=u_{\rho} q=v q / \gamma_{\rho}$, upon increasing interactions.

\section{CONCLUSION}

Using the bosonization technique, we have studied the charge and spin equilibrium dynamics of $1 \mathrm{D}$ fermions in clean quantum wires with forward scattering interactions, i.e., below half filling, and band curvature, i.e., quadratic, corrections to the linear single-particle spectrum [cf. Eq. (3)]. The dynamics were accessed by calculating the charge and magnetic dynamical structure factors at small wave vector $q$. Charge dynamics may be accessed experimentally with the help of energy- and momentum-resolved spectroscopies, ${ }^{28}$ electronic Raman spectroscopy, ${ }^{29}$ or electron energy loss spectroscopies. ${ }^{30}$

The introduction of band-curvature corrections allowed us to explore the high-energy physics of fermions in quantum wires. This a basic step beyond the standard TomonagaLuttinger model which assumes a linear dispersion, Eq. (1).

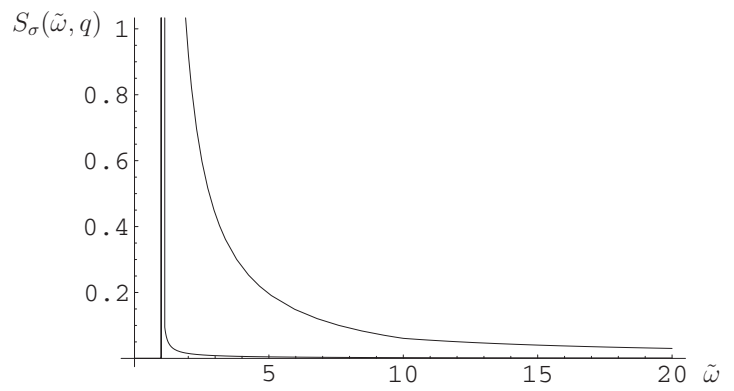

FIG. 14. Magnetic structure factor $S_{\sigma}(\widetilde{\omega}, q)$ at a fixed $q$ as a function of the dimensionless frequency $\widetilde{\omega}=\omega / v q$ and for different interaction strengths in the charge sector $\left(\gamma_{\sigma}=1\right)$. This figure shows a transfer of the spectral weight to higher frequencies as the interaction strength increases. This transfer originates from the spincharge coupling due to band curvature. The spectral weight then extends from the spin excitation frequency $\widetilde{\omega}_{\sigma}=1$ to the interactiondependent charge excitation frequency $\widetilde{\omega}_{\rho}=1 / \gamma_{\rho}$, with additional high frequency tails. The peak at $\widetilde{\omega}=1$ corresponds to the freefermion result $\gamma_{\rho}=1\left(\widetilde{\omega}_{\sigma}=\widetilde{\omega}_{\rho}=1\right)$. The second curve corresponds to the second-order curvature correction at $\gamma_{\rho}=0.9\left(\widetilde{\omega}_{\rho}=1.1\right)$. The third curve corresponds to the second-order curvature correction at $\gamma_{\rho}=0.1\left(\widetilde{\omega}_{\rho}=10\right)$.

In solid-state physics this relativistic approximation is valid only at low energies. It is also a basic step toward the Hubbard model (below half filling) which has the full nonlinear spectrum of lattice fermions, Eq. (2).

Based on recent results for fermions without $\operatorname{spin}^{13,18}$ we have discussed the efficiency and limitations of bosonization in the presence of curvature corrections (see Sec. III). Our main result is Eq. (31) for the self-energy part of the DSF, in second order in band curvature and to all orders in interactions. From this self-energy the two-pair continuum of the DSF has been derived, following Ref. 13, and in agreement with other known results. ${ }^{19,21}$ Moreover, a resummation of band-curvature effects based on the second-order self-energy enabled us to emulate the two-parametric structure of the single-pair continuum [see Eq. (37)], in qualitative agreement with exact results. ${ }^{13,18}$ On the other hand, the precise line shape of the DSF could not be derived with the help of this self-energy and a more efficient resummation scheme has to be developed.

In the case of fermions with spin we have focused on the spin-charge coupling which arises from the interplay between curvature and interactions (see Sec. IV), following Ref. 15. The spin-charge coupling manifests by the opening of new channels for the decay of the excitations. For charge excitations, besides the usual decay into two other chargeneutral excitations, as in the spinless case, a decay into two spin singlets takes place. On the other hand, there is a unique channel of decay of spin (singlet or triplet) excitations: via a pair composed of the spin excitation itself and a chargeneutral excitation. The decay of spin excitations therefore always affects the charge degrees of freedom.

For the charge dynamics our main result is Eq. (45) the self-energy part of the charge DSF, in second order in band curvature and to all orders in interactions. This equation and the corresponding Fig. 11 show that the charge DSF has a double-peak structure: a charge peak at $\omega=u_{\rho}|q|$ but also a 
spin peak at $\omega=v|q|$. The charge peak corresponds to an incoherent continuum of single spinless charge-neutral excitations. It has a high-frequency tail corresponding to the incoherent continuum of pairs of these spinless charge-neutral excitations. On the other hand the spin peak corresponds to the incoherent continuum of chargeless single spin-singlet excitations. A resummation based on the second-order selfenergy has revealed that the two-parametric structure of the charge peak is qualitatively similar to the case of spinless fermions. The width of the spin peak as well as the precise line shape of the charge DSF could not be derived with the help of this self-energy. For the spin dynamics our main result is Eq. (58), the self-energy part of the spin DSF, in second order in band curvature and to all orders in interactions. This self-energy displays a transfer of magnetic spectral weight to higher frequencies. This continuous transfer extends from the spin frequency $\omega=v|q|$ to the charge frequency $\omega=u_{\rho}|q|$ with additional tails at $\omega \gg u_{\rho}|q|$. Because $u_{\rho}=v / \gamma_{\rho}$ is interaction dependent, the transfer of magnetic spectral weight to higher frequencies increases with increasing repulsive interactions (see Fig. 14).

\section{ACKNOWLEDGMENTS}

I thank the Abdus Salam ICTP for hospitality. I am deeply grateful to S. Brazovskii, F. H. L. Essler, and A. A. Nersesyan for fruitful discussions as well as the referees for their criticism and suggestions.

\section{APPENDIX: NOTATIONS}

Our notations are different from the ones often found in the literature (cf. Refs. 5, 13, and 16). They have

$$
\mathcal{H}_{\text {curvature }}=\frac{1}{12 \pi m}\left\{:\left[\partial_{x} \varphi_{R}(x)\right]^{3}:-:\left[\partial_{x} \varphi_{L}(x)\right]^{3}:\right\},
$$

with $\psi_{R(L)}(x) \sim e^{ \pm i k_{F} x-i \varphi_{R(L)}}$. We have therefore the following correspondence between these notations and our notations:

$$
\varphi_{+} \rightarrow-\varphi_{R} / \sqrt{4 \pi}, \quad \varphi_{-} \rightarrow \varphi_{L} / \sqrt{4 \pi} .
$$

We may check that, indeed, this yields the same numerical factor $(2 \sqrt{\pi} / 3 m)(1 / 8 \pi \sqrt{\pi})=1 / 12 \pi m$.

Moreover, the chiral basis $\varphi_{ \pm}$is often used. We wish to relate the general irrelevant operator expressed in the $\varphi$ basis,

$$
\partial_{x} \varphi\left[n\left(\partial_{\tau} \varphi\right)^{2}-v^{2}\left(\partial_{x} \varphi\right)^{2}\right]
$$

to its expression in the chiral basis $\left(n=3 / \gamma_{\rho}^{2}\right.$ in the text). In real time this can be done with the help of $(1 / v) \partial_{t} \varphi=\Pi_{\varphi}=$ $-\partial_{x} \varphi_{+}+\partial_{x} \varphi_{-}$and $\partial_{x} \varphi=\partial_{x} \varphi_{+}+\partial_{x} \varphi_{-}$, and yields

$$
\begin{aligned}
\partial_{x} \varphi[ & \left.n\left(\partial_{\tau} \varphi\right)^{2}-v^{2}\left(\partial_{x} \varphi\right)^{2}\right] \\
= & -(n+1)\left[\left(\partial_{x} \varphi_{+}\right)^{3}+\left(\partial_{x} \varphi_{-}\right)^{3}\right] \\
& \quad+(n-3)\left[\left(\partial_{x} \varphi_{+}\right)^{2} \partial_{x} \varphi_{-}+\partial_{x} \varphi_{+}\left(\partial_{x} \varphi_{-}\right)^{2}\right] .
\end{aligned}
$$

The case $n=3$ corresponds to free fermions:

$$
\begin{aligned}
\partial_{x} \varphi\left[3\left(\partial_{\tau} \varphi\right)^{2}-v^{2}\left(\partial_{x} \varphi\right)^{2}\right] & =-4\left[\left(\partial_{x} \varphi_{+}\right)^{3}+\left(\partial_{x} \varphi_{-}\right)^{3}\right] \\
& =-\frac{1}{\pi}\left[\left(\partial_{x} \varphi_{R}\right)^{3}+\left(\partial_{x} \varphi_{L}\right)^{3}\right],
\end{aligned}
$$

and is the sum of two chiral terms. This implies that the tails are in the crossed chiral terms. They can be isolated by a special (unrealistic) choice of the interactions, $n=-1$, which yields

$$
\begin{aligned}
\partial_{x} \varphi\left[\left(\partial_{\tau} \varphi\right)^{2}+v^{2}\left(\partial_{x} \varphi\right)^{2}\right] & =4\left[\left(\partial_{x} \varphi_{+}\right)^{2} \partial_{x} \varphi_{-}+\partial_{x} \varphi_{+}\left(\partial_{x} \varphi_{-}\right)^{2}\right] \\
& =\frac{1}{\pi \sqrt{4 \pi}}\left[\left(\partial_{x} \varphi_{R}\right)^{2} \partial_{x} \varphi_{L}-\partial_{x} \varphi_{R}\left(\partial_{x} \varphi_{L}\right)^{2}\right],
\end{aligned}
$$

where Eq. (A1) has been used.
*Electronic address: sofian.teber@grenoble.cnrs.fr

${ }^{1}$ S.-I. Tomonaga, Prog. Theor. Phys. 5, 544 (1950).

${ }^{2}$ J. M. Luttinger, J. Math. Phys. 4, 1154 (1963); D. C. Mattis and E. H. Lieb, ibid. 6, 304 (1965).

${ }^{3}$ M. Schick, Phys. Rev. 166, 404 (1968).

${ }^{4}$ A. Luther and V. J. Peschel, Phys. Rev. B 9, 2911 (1974); S. Coleman, Phys. Rev. D 11, 2088 (1975).

${ }^{5}$ F. D. M. Haldane, J. Phys. C 14, 2585 (1981).

${ }^{6}$ T. Giamarchi, Quantum Physics in One Dimension (Oxford University Press, New York, 2004).

${ }^{7}$ A. O. Gogolin, A. A. Nersesyan, and A. M. Tsvelik, Bosonization and Strongly Correlated Systems (Cambridge University Press, Cambridge, U.K., 2004).

${ }^{8}$ D. Pines and P. Nozières, The Theory of Quantum Liquids (Perseus Books, New York, 1989), Vol. 1.

${ }^{9}$ F. H. L. Essler, H. Frahm, F. Göhmann, A. Klümper, and V. E. Korepin, The One-Dimensional Hubbard Model (Cambridge University Press, Cambridge, U.K., 2005).

${ }^{10}$ N. Andrei, in Low-Dimensional Quantum Field Theories for Con- densed Matter Physicists, edited by S. Lundqvist, G. Morandi, and $\mathrm{Yu}$ Lu, Series on Modern Condensed Matter Physics Vol. 6 (World Scientific, Singapore, 1995).

${ }^{11}$ F. H. L. Essler and V. E. Korepin, Phys. Rev. B 59, 1734 (1999).

${ }^{12}$ N. Kitanine et al., arXiv:hep-th/0505006 (unpublished).

${ }^{13}$ R. G. Pereira, J. Sirker, J.-S. Caux, R. Hagemans, J. M. Maillet, S. R. White, and I. Affleck, Phys. Rev. Lett. 96, 257202 (2006).

${ }^{14}$ I. Affleck, in Fields, Strings and Critical Phenomena, edited by E. Brézin and J. Zinn-Justin, Proceedings of the Les Houches Summer School of Theoretical Physics XLIX (Elsevier Science Publishers, Amsterdam, 1990).

${ }^{15}$ S. Brazovskii, S. Matveenko, and P. Nozières, J. Phys. I 4, 571 (1994); S. Brazovskii and S. Matveenko JETP 78, 892 (1994).

${ }^{16}$ K. V. Samokhin, J. Phys.: Condens. Matter 10, L533 (1998).

${ }^{17}$ P. Pirooznia and P. Kopietz, arXiv:cond-mat/0512494 (unpublished).

${ }^{18}$ M. Pustilnik, M. Khodas, A. Kamenev, and L. I. Glazman, Phys. Rev. Lett. 96, 196405 (2006).

${ }^{19}$ M. Pustilnik, E. G. Mishchenko, L. I. Glazman, and A. V. An- 
dreev, Phys. Rev. Lett. 91, 126805 (2003).

${ }^{20}$ A. V. Rozhkov, Eur. Phys. J. B 47, 193 (2005); Phys. Rev. B 74, 245123 (2006).

${ }^{21}$ S. Teber, Eur. Phys. J. B 52, 233 (2006).

${ }^{22}$ A. G. Abanov and P. B. Wiegmann, Phys. Rev. Lett. 95, 076402 (2005).

${ }^{23}$ The equality $\gamma_{i} u_{i}=v$, where $i=\rho, \sigma$, holds for the bare parameters, i.e., without taking into account umklapp and backscattering. When taken into account, these processes renormalize the bare parameters so that the inequality no longer holds. In the present case, umklapp and backscattering are irrelevant so that their effect is weak and, to a good approximation, we may consider that the equality still holds (see Ref. 6).

${ }^{24}$ This agrees with our earlier result, derived in Ref. 21, that band curvature yields subleading corrections to the electron selfenergy: $\operatorname{Im} \Sigma \sim|\epsilon|+|\epsilon|^{3} / \epsilon_{F}^{2}$, where $\epsilon_{F}=m v^{2}$, The leading linear part is a signature that the electrons are not the stable quasiparticles of the system.

${ }^{25}$ This is seen from the phase shifts associated with the scattering between the elementary excitations forming physical ones and determined by the Bethe ansatz solution to the Hubbard model (see Ref. 10). For example, the phase shift associated with the scattering between two spinons forming a singlet has one term describing pure spin scattering and another term, below half filling, describing the scattering of a spinon off an incoherent background charge which is perturbed by the presence of the singlet.

${ }^{26}$ The model of spinful fermions is invariant under the large group $\mathrm{U}(1) \times \mathrm{SU}(2)$, which has a non-Abelian component arising from the spin of the fermions. A rigorous procedure would require using the non-Abelian bosonization technique to treat the spin sector. This would yield the so-called SU(2) level-1 Sugawara Hamiltonian and the corresponding Wess-Zumino-Witten (WZW) Lagrangian (see the textbook Ref. 7), properly generalized to include the effect of curvature. A formulation of the problem within the WZW nonlinear $\sigma$ model is thus possible. In the present study, we restrict ourselves to a simpler Abelian bosonization and, whenever necessary, mention the constraints arising from the $\mathrm{SU}(2)$ symmetry.

${ }^{27}$ In the literature on the Hubbard model both of these peaks are known, e.g., in Ref. 9 see Fig. 7.29 for the particle-hole peak and Fig. 7.31 for the singlet peak (the latter is degenerate with the spin triplet).

${ }^{28}$ O. M. Auslender et al., Science 295, 825 (2002).

${ }^{29}$ F. Perez, B. Jusserand, and B. Etienne, Phys. Rev. B 60, 13310 (1999).

${ }^{30}$ T. Pichler, M. Knupfer, M. S. Golden, J. Fink, A. Rinzler, and R. E. Smalley, Phys. Rev. Lett. 80, 4729 (1998). 